\title{
Endbericht
}

\section{Zwischenevaluierung des Förderprogramms IÖB-Toolbox}

Jürgen Streicher, Marija Breitfuss-Loidl, Michael Ploder JOANNEUM RESEARCH

Eva Buchinger Austrian Institute of Technology

Im Auftrag der Austria Wirtschaftsservice (aws)

Wien/Graz, August 2021 


\title{
1IT T:
}

\section{Endbericht}

\section{Zwischenevaluierung des Förderprogramms IÖB-Toolbox}

\author{
Jürgen Streicher, Marija Breitfuss-Loidl, Michael Ploder
} JOANNEUM RESEARCH

Eva Buchinger
Austrian Institute of Technology

JOANNEUM RESEARCH Forschungsgesellschaft $\mathrm{mbH}$ Institut für Wirtschafts- und Innovationsforschung

\section{Büro Graz}

Leonhardstraße 59 8010 Graz, Austria

Tel.: +43-316-876 1488

E-Mail: policies@joanneum.at
Büro Wien

Haus der Forschung, Sensengasse 1

1090 Wien, Austria

Tel.: +43-1-5817520

E-Mail: policies@joanneum.at
Büro Klagenfurt

Lakeside B08a, EG

9020 Klagenfurt am Wörthersee

Tel.: +43-1-876 7553

E-Mail: policies@joanneum.at 


\section{Inhaltsverzeichnis}

1 EINLEITUNG

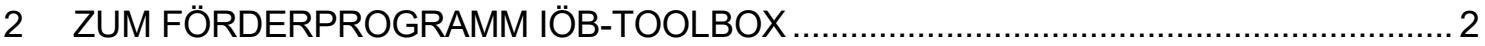

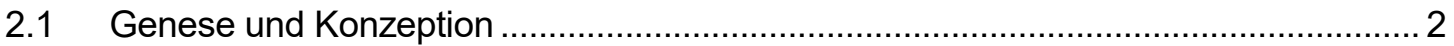

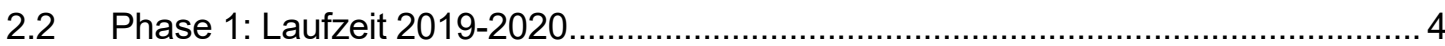

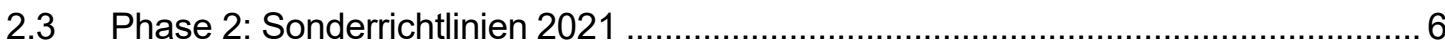

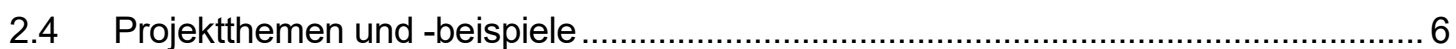

3 IÖB MIT UNTERSTÜTZUNG DER TOOLBOX - EIN EMPIRISCHER BEFUND .............. 9

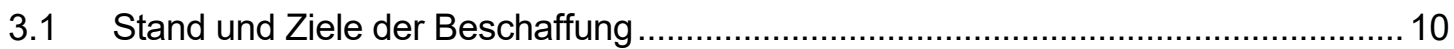

3.2 Ergebnisse und Wirkungen ……......................................................................... 14

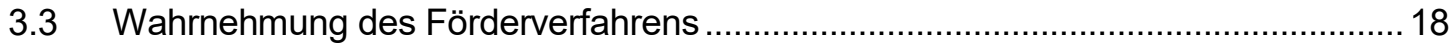

3.4 Verbesserungspotentiale aus Sicht der Befragten ............................................... 19

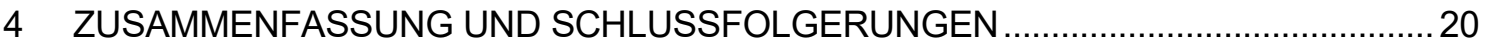

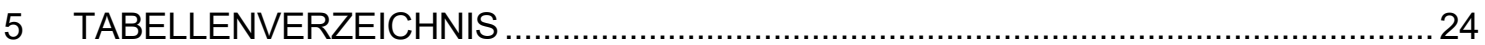

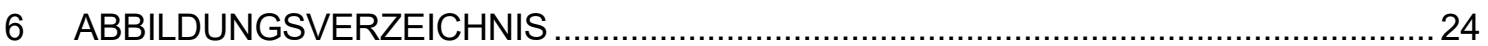




\section{Einleitung}

Innovationsfördernde öffentliche Beschaffung (IÖB) ist ein wichtiger Eckpfeiler der nachfrageseitigen Innovationspolitik. IÖB soll dabei helfen, neue Märkte für Innovationen zu schaffen und die Nachfrage nach neuen, gesellschaftsrelevanten, innovativen Gütern und Dienstleistungen zu erhöhen. Das hohe Volumen öffentlicher Beschaffung verspricht eine aus innovationspolitischer Sicht bedeutende Hebelwirkung, wichtig ist auch die Vorbildfunktion und -wirkung. Zudem kann der öffentliche Sektor als Referenzmarkt für in Österreich entwickelte neue Technologien und Dienstleistungen dienen.

IÖB ist in Österreich seit 2007 Thema und seit 2012 mit dem beschlossenen und seit damals schrittweise umgesetzten „IÖB Leitkonzept“ politisch verankert. Zu den wichtigsten Meilensteinen auf organisatorischer Ebene zählt die Etablierung eines IÖB-Service Netzwerkes mit der IÖB-Servicestelle als Knotenpunkt und komplementären IÖB-Kompetenz- und Kontaktstellen. Dazu finden sich eine Reihe von IÖB-Pilot- und Referenzprojekten sowie Instrumente zur Unterstützung der Umsetzung von IÖB, wie etwa die IÖB-Challenges oder der IÖB-Marktplatz. Zu den jüngsten Fördermaßnahmen zählt die IÖB-Toolbox. Das Programm wurde 2018 von der aws in ihrer Funktion als IÖB-Kompetenzstelle entwickelt, unterstützt durch das Bundesministerium für Klimaschutz, Umwelt, Energie, Mobilität, Innovation und Technologie (BMK) und das Bundesministerium für Digitalisierung und Wirtschaftsstandort (BMDW). Die Laufzeit betrug zunächst zwei Jahre und wurde mit Mitteln der Nationalstiftung (Österreich-Fonds) durchgeführt (Phase 1: 2019-2020). Seit 2021 wird die IÖB-Toolbox im Rahmen der IÖBInitiative aus Mitteln des BMK dotiert und weitergeführt (Phase 2). Die Abwicklung erfolgt in beiden Phasen durch die aws.

Ziel der Evaluierung ist es, eine Zwischenbewertung der Umsetzung des Programms IÖBToolbox in der ersten Phase (2019-2020) sowie der bis dato feststellbaren Beiträge zu Zielerreichung und ggf. erster Wirkungen vorzunehmen. Dabei wird auch ein Schlaglicht auf die Konzeption des Förderprogramms geworfen. Gemäß Ausschreibung liegt der Fokus der Evaluierung auf der Konzeption und Durchführung einer Befragung der Förderungsnehmerlnnen (öffentliche BeschafferInnen) sowie deren LieferantInnen im konkreten Projekt. Auf dieser Basis wurden Schlussfolgerungen und Handlungsoptionen für die Weiterführung des Programms abgeleitet. Mit Blick auf die Befragungen standen folgende Fragen im Vordergrund:

- Welche Ziele wurden mit dem innovativen Beschaffungsvorhaben verfolgt, welche Herausforderungen gab es bei der Umsetzung?

- Was waren die beobachtbaren Wirkungen bei dem/der Förderungsnehmerln und deren LieferantInnen? Wie lassen sich diese beschreiben?

- Wie wird die Förderung insgesamt und deren Abwicklung bewertet?

Die Durchführung der Evaluierung erfolgte in einem relativ kurzen Zeitraum (Mai-Juli 2021). Den Ausgangspunkt bildeten ein Kick-Off mit dem Auftraggeber aws sowie eine Sichtung und Analyse von relevanten Dokumenten und verfügbaren Daten. Dazu zählten neben den Programmdokumenten der ersten Phase $(2019,2020)$ und der Sonderrichtlinien von 2021 vor allem die Berichte der aws an die Nationalstiftung $(2019,2020)$ sowie die Förderstatistik der aws. Um die gewonnen Erkenntnisse aus der Dokumentenanalyse zu überprüfen sowie Erfah- 
rungen mit der Genese und Umsetzung des Förderprogramms einzuholen, wurden leitfadengestützte Interviews mit VertreterInnen der IÖB-Ressorts (BMK, BMDW), des Programmmanagements der aws sowie der IÖB-Servicestelle durchgeführt.

Insgesamt wurden drei Online-Befragungen konzipiert und durchgeführt. Eine Befragung richtete sich an die Förderungsnehmerlnnen des Fördermoduls Transfer, eine an die Förderungsnehmerlnnen des Fördermoduls Prepare und eine an die Lieferantlnnen der FörderungsnehmerInnen des Fördermoduls Transfer. ${ }^{1}$ Darüber hinaus wurden mit drei externen ExpertInnen im Bereich innovationsfördernder öffentlicher Beschaffung Gespräche geführt, darunter jene BeraterInnen, die die Gestaltung und Durchführung der IÖB-Challenges im Modul Prepare begleiteten.

\section{Zum Förderprogramm IÖB-Toolbox}

Mit dem IÖB-Leitkonzept von 2012 bzw. der Umsetzung der darin angelegten Maßnahmen konnten in Österreich viele Akzente bei der Erhöhung des Anteils an innovativen und innovationsfördernden Beschaffungen an der Gesamtheit der öffentlichen Beschaffung gesetzt werden. ${ }^{2}$ Die Potentiale nachfrageorientierter Innovationspolitik werden gegenwärtig aber noch nicht ausgeschöpft. Die Schaffung und Weiterentwicklung von geeigneten Anreizsystemen soll die Übernahme von Risiken begünstigen und die Bereitschaft zur Teilnahme von öffentlichen BeschafferInnen an Innovationsbeschaffung bzw. den damit verbundenen Prozessen erhöhen.

\subsection{Genese und Konzeption}

Die IÖB-Toolbox wurde als Nachfolger des IÖB-Projektwettbewerbs entwickelt. Im Rahmen dieser Maßnahme konnten öffentliche BeschafferInnen zwischen 2013 und 2017 finanzielle Unterstützung für innovative Beschaffungsprojekte gewinnen. Der Projektwettbewerb wurde als positiv beurteilt und für eine Weiterführung empfohlen. ${ }^{3}$ Besonders die „doppelten Wirkungen" eines Förderinstruments sowie eines Instruments zur Sichtbarmachung erfolgreicher IÖBAnsätze wurden hervorgehoben. Im Falle einer Weiterentwicklung des Projektwettbewerbs sollte eine der Förderagenturen, wie in der IÖB-Toolbox umgesetzt, die Abwicklung übernehmen um die Aufgaben- und Rollenteilung im IÖB-Service Netzwerkes effizienter zu gestalten und die IÖB-Servicestelle zu entlasten.

Laut den im Rahmen dieser Untersuchung geführten Interviews wurde überlegt, das Format von der Abgeltung der Kosten zur Durchführung eines innovativen Beschaffungsprojekts hin zu einer direkten Förderung der Beschaffung eines konkreten Produkts oder einer Dienstleistung weiterzuentwickeln. Das Instrument sollte öffentliche Beschafferlnnen mit einem konkreten Bedarf ansprechen. Zudem wurde nach einem Ansatz gesucht, die Beschafferlnnen bei

\footnotetext{
${ }^{1}$ Die Fördermodule werden in Abschnitt 2.1 kurz beschrieben.

${ }^{2}$ Vgl. Ruhland, Sascha und Kaufmann, Joachim und Ploder, Michael und Zinke, Guido (2018): Evaluierung der Umsetzung des Leitkonzepts für eine innovationsfördernde öffentliche Beschaffung in Österreich. Endbericht. Technischer Bericht. Wien.

${ }^{3}$ Vgl. ebda.
} 
der Recherche nach einer optimalen Lösung zu unterstützen und damit auch verstärkt IÖBChallenges ${ }^{4}$ anzustoßen.

Das Konzept für das neue Format „IÖB-Toolbox“ wurde 2018 von der aws gemeinsam mit den für IÖB zuständigen Ministerien (BMK, BMDW) erarbeitet. Es wurde ein Antrag auf Mittel aus der Nationalstiftung für die Durchführung eingebracht, der positiv beschieden wurde. Für das Programm wurden $€ 1,8$ Mio. für den Zeitraum 2019 bis 2020 (Phase 1) zur Verfügung gestellt.

Das übergreifende Ziel der IÖB-Toolbox ist es, einen Beitrag zur IÖB-Initiative durch die Förderung innovativer Beschaffungsprojekte („Modul Transfer“) bzw. von Beratung zur Umsetzung künftiger innovativer Beschaffungsprojekte („Modul Prepare“) zu leisten. Wie den Programmdokumenten sowie den Interviews zu entnehmen ist, soll durch die Förderung die Sichtbarkeit von IÖB im Allgemeinen erhöht, die Anzahl und Qualität innovativer Beschaffungsvorhaben gesteigert und somit „Vorzeigeprojekte“ bzw. „Good-Practices“ geschaffen werden. Für öffentliche BeschafferInnen sollen Anreize geschaffen, Risiken abgemildert sowie Entscheidungsprozesse beschleunigt und unterstützt werden. Die Beschaffung und der Einsatz von Innovation sollen positive Wirkungen im öffentlichen Sektor (nachfrageseitig) sowie durch die Innovationsnachfrage aus dem öffentlichen Sektor auch positive Wirkungen auf österreichische Unternehmen (angebotsseitig; als AnbieterInnen bzw. LieferantInnen von Innovation) entfalten.

- Transfer: Förderbar sind sowohl die Anschaffung von innovativen Produkten als auch von innovativen Dienstleistungen oder Kombinationen aus Produkt und Dienstleistung. Die maximale Zuschusshöhe beträgt 50 \% (bis April 2019) bzw. 100 \% (ab April 2019; Erhöhung aufgrund der COVID-19-Pandemie) der förderbaren Projektkosten und maximal $100.000 €$ pro Projekt.

- Prepare: Gefördert werden Beratungsleistungen durch externe ExpertInnen, um im Vorfeld innovativer Beschaffungsprojekte die Markterkundung mittels Nutzung des OpenInnovation-Ansatzes (IÖB-Challenge) zu unterstützen. Die Förderungshöhe kann bis zu $100 \%$ der förderbaren Projektkosten, jedoch maximal $15.000 €$ pro Projekt betragen. Der/die Expertln muss über nachweisbare Erfahrungen im Bereich der öffentlichen Beschaffung oder über ein IÖB-Zertifikat verfügen.

Die Förderung wurde in der Form nicht-rückzahlbarer Zuschüsse vergeben. Einreichberechtigt waren alle österreichischen öffentlichen AuftraggeberInnen, die dem Bundesvergabegesetz unterliegen (§3 sowie §164 und §165 BVergG) - mit Ausnahme von Institutionen der österreichischen Bundesverwaltung. Die Förderung wurde als laufende Einreichung mit quartalsweisen Entscheidungsterminen abgewickelt. Zwischen April 2019 (zwei) und September 2020 (vier) fanden insgesamt sechs Jurysitzungen statt.

Die Auswahl der geförderten Projekte erfolgte in einem zweistufigen Verfahren: Im ersten Schritt fand eine Formalprüfung der Anträge statt. Im zweiten Schritt bewertet eine Jury die Projekte für beide Module auf Basis von inhaltlichen Kriterien und gibt eine Förderungsemp-

\footnotetext{
${ }^{4}$ Die IÖB-Challenge ist ein Online-Instrument, mit dem ein öffentlicher Auftraggeber eine Problemstellung auf der IÖBInnovationsplattform ausruft. Unternehmen werden mit dieser Challenge aufgefordert, ihre innovativen Ideen und kreativen Lösungsvorschläge online einzureichen. Die IÖB-Challenge unterstützt den öffentlichen Auftraggeber bei der Marktrecherche und folgt methodisch dem Open Innovation Ansatz. (Quelle: aws)
} 
fehlung ab. Zu den inhaltlichen Kriterien zählten der Innovationsgrad, Aspekte des gesellschaftlichen Mehrwerts und der Effizienzsteigerung sowie der Übertragbarkeit. Die Förderungsentscheidung traf die aws auf Basis der Förderungsempfehlung der Jury.

\subsection{Phase 1: Laufzeit 2019-2020}

Eine erstmalige Einreichung war im April 2019 möglich. Im Gesamtjahr 2019 wurden acht Anträge eingereicht, davon wurden sieben Projekte (sechs Transfer, ein Prepare) positiv entschieden und ein (Prepare-)Projekt aus formalen Gründen abgelehnt (vgl. Tab. 1). Im Jahr 2020 wurden insgesamt 29 Anträge eingereicht, davon wurden 23 Projekte (21 Transfer, zwei Prepare) positiv entschieden und sechs Projekte aufgrund der Juryempfehlung abgelehnt. Mit der Jurysitzung im September 2020 war das Budget ausgeschöpft und die Einreichung wurde geschlossen.

Insgesamt entfiel der Großteil der Anträge auf das Modul Transfer (32). Fünf Einreichungen wurden für das Modul Prepare registriert. 27 Projekte wurden im Modul Transfer und drei im Modul Prepare positiv entschieden. Fünf Transfer-Projekte haben sich trotz zugesagter Förderung gegen eine Projektumsetzung entschieden (,beendet/verzichtet“). Von den durchgeführten 22 Transfer-Projekten befanden sich im Juli 202118 in der Umsetzung. Vier waren abgeschlossen und erhielten den Zuschuss ausbezahlt. Von den drei Prepare-Projekten befanden sich zwei Vorhaben in der Umsetzung. Eines war abgeschlossen bzw. ausbezahlt.

Tabelle 1 Förderstatistik IÖB-Toolbox

\begin{tabular}{|lccccccccc|}
\hline & \multicolumn{3}{c}{$\mathbf{2 0 1 9}$} & \multicolumn{4}{c}{$\mathbf{2 0 2 0}$} & \multicolumn{3}{c|}{ Gesamt } \\
Transfer & Anträge & Zusagen & $\%$ & Anträge & Zusagen & $\%$ & Anträge & Zusagen & $\%$ \\
Prepare & 6 & 6 & 100,0 & 26 & 21 & 80,8 & 32 & $27\left(22^{\star}\right)$ & 84,4 \\
\hline Summe & 2 & 1 & 50,0 & 3 & 2 & 66,7 & 5 & 3 & 60,0 \\
\hline
\end{tabular}

* Fünf Transfer-Projekte haben die zugesagte Förderung nicht in Anspruch genommen („,beendet/verzichtet“). Quelle: aws.

Den 22 durchgeführten Transfer-Projekten mit Gesamtkosten in der Höhe von $€$ 4.192.199 wurden Fördermitteln in der Höhe von $€ 1,61$ Mio. zugeteilt (vgl. Tab. 2). Umgelegt auf die Anzahl der Transfer-Projekte beträgt die durchschnittliche Höhe der Projektkosten $€ 190.555$, die durchschnittliche Höhe des Zuschusses $€$ 73.376. Auf die drei durchgeführten PrepareProjekte entfiel ein Zuschuss von jeweils in etwa dem Maximum der möglichen Förderhöhe $(€ 14.900 /$ Projekt). Die Zuschussquote (gemessen an den förderbaren Projektkosten) lag 2019/2020 insgesamt im Schnitt bei 53 \% (Median).

Projekte wurden mit Ausnahme von Vorarlberg und Salzburg in allen Bundesländern unterstützt. Die durchschnittlichen Projektkosten der geförderten Projekte pro Bundesland bewegte sich zwischen $€ 250.000$ (Wien) und $€ 75.000$ (Steiermark). Laut Interviews mit aws und IÖBServicestelle fanden sich im Modul Transfer viele neue, in der IÖB-Community bisher weniger aktive öffentliche Beschafferlnnen aus dem kommunalen Bereich. 
Tabelle $2 \quad$ Kennzahlen IÖB-Toolbox

\begin{tabular}{|lccc|}
\hline & Gesamtprojektkosten (in $€$ ) & Zuschusshöhe (in $€$ ) & Zuschuss (in \%) \\
2019 & $1.657 .329,1$ & $397.106,0$ & 47,4 \\
2020 & $2.579 .569,6$ & $1.261 .869,0$ & 61,7 \\
\hline Summe & $4.236 .898,8$ & $1.658 .975,0$ & $57,7^{\star *} / 53,3^{\star \star *}$ \\
$\ldots$ Transfer & $4.192 .198,8$ & $1.614 .275,0$ & 51,9 \\
... Prepare & $44.700,0$ & $44.700,0$ & 100,0 \\
\hline
\end{tabular}

* Durchschnitte der Zuschussquoten aller Projekte. ${ }^{* *}$ Arithmetischer Mittelwert. *** Median.

Quelle: aws.

Die Fördermaßnahme blieb 2019 zunächst deutlich hinter den Erwartungen. In einem Interview wurde angemerkt, dass Budgets öffentlicher Organisationen und Einrichtungen oft mit Jahresbeginn fixiert und damit für neue, unterjährige Projekte nur wenig Spielraum bleibt. Zudem war das Förderprogramm beim Start nahezu unbekannt. Dementsprechend wurden mit Blick auf 2020 zusätzliche Maßnahmen gesetzt, um das Programm bekannter zu machen und die Nachfrage zu steigern. Dazu zählten einerseits ein verstärktes Bewerben der Maßnahme über die Netzwerkaktivitäten des IÖB-Service Netzwerkes, allen voran der IÖB-Servicestelle, und Informationsarbeit der aws. Im Laufe der Zeit fanden, wie die aws beobachten konnte, öffentliche Beschafferlnnen verstärkt über Empfehlungen anderer öffentlicher AuftraggeberInnen zum Förderprogramm. Damit potentielle Förderungsnehmerlnnen ihre Anträge gezielt vorbereiten können, wurden für 2020 Cut-off Dates festgelegt und diese gemeinsam mit den Juryterminen auf der aws Webseite bekanntgegeben.

Während sich die gestiegene Nachfrage 2020 einerseits mit der verstärkten Bewerbung und den Empfehlungen in der Community erklären lässt, wurde der COVID-19-Pandemie in den Interviews ein gewisser Einfluss auf das Interesse am Förderprogramm zugeschrieben. Einerseits hat die Pandemie in vielen Bereichen Lücken aufzeigt und Ideen angestoßen. Insbesondere Digitalisierungsbestrebungen wurden im öffentlichen Bereich verstärkt und unterstützt. Die IÖB-Toolbox, vor allem das Modul Transfer, stellte eine willkommene Gelegenheit dar, um Modernisierungsprojekte mitzufinanzieren und zu beschleunigen.

Andererseits stieg mit der Dauer der Pandemie auch die Unsicherheit. Einige Gemeinden dürften aufgrund unerwarteter Aufwendungen, etwa wegen Schutzmaßnahmen zur Eindämmung der Pandemie, weniger Mittel und Ressourcen zur Verfügung haben, um sich entsprechenden Projekten widmen zu können. In den Interviews mit aws wurde von Fällen berichtet, in welchen Anträge zurückgezogen werden mussten, da die Finanzierung des Projekts (doch) nicht sichergestellt werden konnte. Einige der positiv beschiedenen Projekte konnten aufgrund der Pandemie nicht rechtzeitig fertiggestellt werden und wurden verlängert.

Während für das Modul Transfer keine Zielgröße bezüglich der Zahl zu fördernder Projekte festgelegt wurde, wurde für das Modul Prepare von Seiten des Programmmanagements die Umsetzung von drei Challenges anvisiert. Die Zielgröße wurde vor dem Hintergrund festgelegt, dass die für das Modul erforderlichen externen Beraterlnnen zunächst identifiziert und angesprochen und damit ein entsprechendes Netzwerk aufgebaut werden mussten. Voraussetzung für die Übernahme einer Beratungstätigkeit in Prepare war ein „IÖB-Zertifikat“, das die Beraterlnnen im Rahmen einer (kostenlosen) Schulung durch die IÖB-Servicestelle erwerben können. Der Aufbau und die Pflege dieses Beraterlnnen-Netzwerks, das dem Gespräch mit der IÖB-Servicestelle zufolge auch als „Informations- und Werbe-Netzwerk“ für die IÖB-Toolbox 
fungiert, ist ein wesentlicher Nebeneffekt des Förderprogramms. Schließlich verging noch etwas Zeit, bis interessierte (potentielle) Beschafferlnnen gefunden wurden und sich passende Projektideen herauskristallisierten.

\subsection{Phase 2: Sonderrichtlinien 2021}

Nachdem die Mittel der Nationalstiftung im September 2020 zur Gänze ausgeschöpft waren, wird das Programm IÖB-Toolbox seit 2021 im Rahmen der IÖB-Initiative aus Mitteln des BMK weitergeführt. ${ }^{5}$ Abseits der gestiegenen Nachfrage wurde dieser Schritt mit den positiven Eindrücken aus den laufenden Projekten sowie der Schaffung von Kontinuität auf Basis der nunmehr besseren Bekanntheit und Sichtbarkeit in der IÖB-Community begründet. Im Zuge der Weiterführung kann auf ein erfahrenes und eingespieltes Team bestehend aus den IÖBRessorts, der aws und der IÖB-Servicestelle zurückgegriffen werden, dessen Zusammenarbeit von allen Beteiligten als eng und produktiv beschrieben wird.

Wichtigste Änderungen in den Sonderrichtlinien von 2021 betreffen die Rolle des BMK (trifft nun die Förderentscheidung) und der aws (bleibt Abwicklungsstelle), die Zusammensetzung der Jury (neue ExpertInnen, höherer Frauenanteil) sowie den Fokus auf Projekte mit zu erwartenden Beiträgen zum Umwelt- und Klimaschutz, Schonung natürlicher Ressourcen und zur Digitalisierung im öffentlichen Sektor. Zudem wurden Wirkungsindikatoren zur Beurteilung der Zielerreichung im Programmdokument eingeführt.

\subsection{Projektthemen und -beispiele}

Im Programmverlauf 2019-2020 war Digitalisierung bestimmendes Thema der eingereichten Anträge. Laut Einschätzung der aws wies die überwiegende Anzahl der geförderten Projekte eine hohe bis sehr hohe Digitalisierungsintensität auf. Darunter auch viele Projekte mit hohem Beitrag zum Umwelt- und Klimaschutz sowie Projekte, die Aspekte von Nachhaltigkeit und deren Umsetzung adressieren (Grüne Technologien [,Green Tech"]; unterstützende, nachhaltige digitale Technologien [,tech4green“]). Nachfolgende Beispiele illustrieren und beschreiben innovative Beschaffungsvorhaben, die mit der IÖB-Toolbox gefördert wurden:

\section{DSB-Digitale Sitzungen in der Blockchain ${ }^{6}$ (Transfer)}

Die COVID-19-Pandemie hat den Bedarf an digitalen Kollaborations-und (offiziellen) Sitzungslösungen deutlich gemacht und beschleunigt. Dieser Bedarf konnte allerdings mit einfachen Web-Konferenztools, die die Kommunikation unterstützen, nur unzureichend abgedeckt werden. Bedenken in punkto Cyber-Sicherheit, Datenschutz und ordnungsgemäßer Dokumentation behindern die virtuelle Handlungsfähigkeit von privaten und öffentlichen Rechtsträgern. Gründe, die zu einer bestimmten Beschlussfassung geführt haben, sind nachträglich oft nur schwer nachvollziehbar. Die Rechtssicherheit und die Beweiskraft von Beschlussfassungen

\footnotetext{
${ }^{5}$ IÖB-Toolbox. Sonderrichtlinien der Bundesministerin für Klimaschutz, Umwelt, Energie, Mobilität, Innovation und Technologie im Einvernehmen mit dem Bundesminister für Finanzen auf Basis der Allgemeinen Rahmenrichtlinien für die Gewährung von Förderungen aus Bundesmitteln (ARR 2014). Wien, Dezember 2020.

${ }^{6}$ https://dih-ost.at/product/sicher-onlinesitzung-in-der-blockchain/
} 
leiden darunter massiv. Ebenso werden dadurch den Bürgerlnnen entscheidende Informationen vorenthalten, an deren Offenlegung sie bei öffentlich-rechtlichen Beschlussfassungen ein berechtigtes Interesse hätten.

Im Rahmen des geförderten Projekts wurde ein Pilot für den Einsatz eines virtuellen SitzungsTools entwickelt, mit den Gemeinden, Vereine und Organisationen zukünftig digitale Sitzungen rechtsgültig und nachvollziehbar absolvieren können. Durch Einbeziehung entsprechender Stakeholder konnte erfolgreich überprüft werden, wie ein derartiges Tool funktionieren muss und ob es im Echtbetrieb auch angenommen und verwendet wird. Durch den Piloten wurde nicht nur ein hohes Maß an Committment erreicht, sondern auch die weitere Produktentwicklung erheblich unterstützt. Von Seiten der Projektbeteiligten wird erwartet, dass das Projekt erheblichen Einfluss auf den Einsatz dieser Technologie im Gemeinde- und Vereinsbereich in Österreich haben wird.

\section{Prozessautomatisierung für das Unternehmenscontrolling ${ }^{7}$ (Transfer)}

Robotergesteuerte Prozessautomatisierung (Robotic Process Automation, RPA) ist ein Ansatz zur automatisierten Bearbeitung von strukturierten Geschäftsprozessen durch digitale Software-Roboter (RPA-Bots). Diese übernehmen die Rollen und Aufgaben von Anwenderlnnen und interagieren mit anderen Softwaresystemen. viadonau Österreichische WasserstraßenGesellschaft $\mathrm{mbH}$ verfügt über ein umfassendes Prozessmanagementsystem und prüft kontinuierlich mögliche Verbesserungen der Prozesse und IT-Systeme. 2018 erfolgte eine erste Auseinandersetzung mit der RPA-Technologie, weitere Potentialanalysen folgten. Die IÖBServicestelle unterstützte viadonau zunächst dabei, den Dialog mit RPA-Software-Anbietern zu forcieren.

Die Summe der internen Vorarbeiten mündete in der Entscheidung, ein Proof-of-Concept durchzuführen. Anfang 2020 startete viadonau mit einem Controllingprozess als „early adopter". Im März 2020 wurde ein funktionsfähiger RPA-Bot zur Generierung und Bereitstellung von umfassenden Auswertungen der betrieblichen Ressourcenplanung (Enterprise Resource Planning, ERP) für das quartalsweise Reporting dem Unternehmenscontrolling übergeben. Das Projekt wurde mittels Direktvergabe umgesetzt. Der Pilot schloss mit einem Konzept zur unternehmensweiten Ausrollung ab, dessen Umsetzung durch die IÖB-Toolbox gefördert wurde. Dabei wurde die RPA-Software angepasst, ein internes RPA-Competence Center aufgebaut und weitere unternehmensinterne Prozesse automatisiert. Im Jahr 2020 betrug die Nettoentlastung rd. $€$ 17.000. Für die Folgejahre wird mit jährlichen Einsparungen in der Höhe von etwa $€ 90.000$ gerechnet. Das Projekt wurde 2021 mit dem Sonderpreis des Österreichischen Verwaltungspreises 2021 für innovationsfördernde öffentliche Beschaffung ausgezeichnet.

\section{E-Mobil Naturpark Pöllauer Tal ${ }^{8}$ (Transfer)}

Die im Naturpark Pöllauer Tal gelegene Marktgemeinde Pöllau ist kaum durch öffentliche Verkehrsmittel erschlossen. Die Gemeinde ist stark durch motorisierten Individualverkehr geprägt. Zudem entstehen aufgrund von Zersiedelung und der demografischen Entwicklung, welche

\footnotetext{
${ }^{7}$ https://www.ioeb.at/fileadmin/ioeb/Dokumente/Infothek/IOEB Best Practice viadonau n.pdf

${ }^{8}$ https://www.naturpark-poellauertal.at/news/gemeinsam-elektrisch-unterwegs-im-naturpark-poellauer-tal/
} 
sich in einem steigenden Anteil der älteren und einem sinkenden Anteil der jüngeren Generation ausdrückt, neue Anforderungen an Mobilität und Verkehr. Die seit 2012 existierende, ehrenamtliche Mobilitätsinitiative „mobil50plus“- private Fahrer mit eigenem PKW - bietet Menschen in der Region ohne eigenen Pkw die Möglichkeit, tägliche Aktivitäten wie Einkäufe, Behördenwege, Arztbesuche etc. zum Selbstkostenpreis zu erledigen. Fahrtenwünsche müssen jedoch frühzeitig und telefonisch angemeldet werden. Das Angebot stieß mit der Zeit an seine Grenzen, was die Gemeinde zum Ausgangspunkt für neue Überlegungen mit Blick auf Nachbarschaftshilfe und klimaschonende Mobilität nahm.

Von der Marktgemeinde Pöllau wurde ein Elektrofahrzeug angeschafft und dem Verein zur Verfügung gestellt. Das Fahrzeug soll zum einen den bestehenden Mobilitätsdienst ergänzen, aber auch einer größeren Gruppe als Carsharing zur Verfügung gestellt werden. Hierfür wurde, unterstützt von der IÖB-Toolbox, ein Online-Fahrtenreservierungs- und Kommunikationstool angeschafft, das auch via App am Smartphone aufgerufen werden kann. Einerseits kann dadurch beispielsweise das Elektrofahrzeug digital gebucht und später kontaktlos in Betrieb genommen werden. Andererseits wird ein Überblick über die Fahrzeug- und FahrerInnenverfügbarkeit des Mobilitätsdienstes geboten, die ebenfalls über das Online-Tool gebucht werden können. Neben der Erweiterung des Pools an verfügbaren Fahrzeugen und der Aufwandsreduzierung durch den digitalen Buchungsprozess trägt das Projekt laut Beteiligten zu einer Bewusstseinsbildung und zur Förderung nachhaltiger Mobilität sowie zu einer Stärkung der örtlichen Wirtschaft und sozialer Kontakte bei.

\section{Optimierung von Windkraftanlagen ${ }^{9}$ (Prepare)}

Energie Burgenland ist Österreichs größter Windstromproduzent. In 19 Ortschaften mit 225 Windenergieanlagen und einer Leistung von insgesamt $522 \mathrm{MW}$ werden jährlich rd. $1 \mathrm{Mrd}$. kWh Ökostrom produziert. Gleichzeitig produzieren all diese Windenergieanlagen täglich mehrere Tausend Datenpunkte, die zur Optimierung und zum Vergleich der Erträge verschiedener Anlagen genutzt werden können. Um das Potential dieser bereits bis zu 20 Jahren gesammelten Datensätze zu nutzen, suchte Energie Burgenland nach Partnerlnnen mit dem notwendigen Know-How in der Analyse von Big Data und Anwendungen der Erkenntnisse zur Projekterfahrung, bevorzugt im Energiebereich bzw. Windkraftbereich.

Durch eine Zusammenarbeit mit externen Unternehmen erhofft sich die Energie Burgenland einerseits die Gesamtperformance des Windparks zu erhöhen und andererseits Windenergiekundlnnen neue digitale Services anbieten zu können. Zusätzlich soll das externe Know-How auch Mitarbeitern aus der Windabteilung neue Impulse und Ideen für die Weiterentwicklung des bestehenden Angebots bieten. Für die IÖB-Challenge wurden folgende Fragen formuliert: Wie kann Energie Burgenland riesige Datenmengen von Windenergieanlagen nutzen, um die Performance dieser zu steigern? Welche Datenanalyseverfahren können genutzt werden, um das beste Ergebnis zu garantieren?

Im Zuge der IÖB-Challenge erfolgte eine Vorauswahl durch einen Expertenkreis der Energie Burgenland, um die Top 5 Konzepte zu einem Innovationsdialog einzuladen. Bei diesem Innovationsdialog hatten die EinreicherInnen die Möglichkeit, ihr Konzept zu präsentieren. Energie

\footnotetext{
${ }^{9}$ https://www.energieburgenland.at/unternehmen/presse/mediathek/presseaussendungen/energie-burgenland-startetnaechste-etappe-ihrer-internationalen-start-up-challenge.html
} 
Burgenland erhält mit dieser Challenge einen Überblick über Lösungswege und potentielle PartnerInnen. Es ist geplant, mit einem Einreicher/einer Einreicherin ein Pilotprojekt durchzuführen, um die Windkraftanlagen der Energie Burgenland noch effizienter zu machen.

\section{IÖB mit Unterstützung der Toolbox - Ein empirischer Befund}

Kernstück der Zwischenevaluierung bilden die Befragungen der Förderungsnehmerlnnen im Modul Transfer, deren LieferantInnen sowie die Prepare-FördernehmerInnen der ersten Programmphase (2019-2020). Letztere wurden, trotz der geringen Anzahl, aus Kosten-/Nutzenerwägungen und aufgrund von Schnittmengen mit der Befragung der Transfer-Förderungsnehmerlnnen ebenfalls online befragt. Die Kontaktdaten wurden von der aws zur Verfügung gestellt, darunter auch jene der LieferantInnen, die seitens der aws über die Förderungsnehmerlnnen erhoben wurde. Laufzeit der drei Befragungen war von 21. Juni bis 16. Juli 2021.

In die Online-Befragung der FörderungsnehmerInnen im Modul Transfer wurden insgesamt 22 positiv beschiedene bzw. durchgeführte Anträge eingebunden. Im Falle von Modul Prepare waren es drei Anträge. Für die Befragung der LieferantInnen der öffentlichen BeschafferInnen im Modul Transfer wurden zehn Kontakte zur Verfügung gestellt. ${ }^{10} \mathrm{Im}$ Zuge einer telefonischen Nachrecherche stellte sich heraus, dass zwei Transfer-Projekte die zugesagte Förderung voraussichtlich nicht in Anspruch nehmen werden. Diese wurden für die weiteren Erhebungen nicht mehr kontaktiert.

Insgesamt haben von den letztlich 20 Förderungsnehmerlnnen im Modul Transfer 15 an der Befragung teilgenommen (Rücklaufquote: 75 \%; vgl. Tab. 3). Im Falle von Modul Prepare haben die VertreterInnen aller drei bewilligten Projekte den Fragebogen ausgefüllt. Von den LieferantInnen der öffentlichen BeschafferInnen im Modul Transfer nahmen fünf von zehn Unternehmen teil (50\%).

Tabelle 3 Online-Befragung: Übersicht Datenbasis und Rücklauf

\begin{tabular}{|lcccc|}
\hline & \multicolumn{2}{c}{$\begin{array}{c}\text { Transfer, } \\
\text { Beschaffer }\end{array}$} & $\begin{array}{c}\text { Transfer, } \\
\text { Lieferanten }\end{array}$ & $\begin{array}{c}\text { Prepare, } \\
\text { Beschaffer* }\end{array}$ \\
\hline Verwendete Datenbasis & 22 & $100 \%$ & 10 & 3 \\
\hline ... Beendet / verzichtet It. Auskunft & 2 & $9,1 \%$ & & - \\
Nutzbare Datenbasis & 20 & - & - & - \\
... Nicht geantwortet, Absagen & 5 & $22,7 \%$ & 5 & 3 \\
... Teilgenommen & 15 & $68,2 \%$ & 5 & $100 \%$ \\
\hline Rücklaufquote (im Verhältnis zur nutzbaren Datenbasis) & & $75 \%$ & $50 \%$ & - \\
\hline
\end{tabular}

* Potentielle Nachfrager, IÖB-Challenge Sponsoren

\footnotetext{
${ }^{10}$ Die geringe Anzahl lässt sich dadurch erklären, dass sich einige der Projekte im Modul Transfer in der Vorbereitung- oder frühen Umsetzungsphase befinden. Lieferanten wurden entweder noch nicht ausgewählt oder die bestellte Lösung noch nicht im ausreichenden Maße fertiggestellt.
} 
Die folgenden Ausführungen beruhen auf den drei im Rahmen der Evaluierungen durchgeführten Befragungen. Zusätzlich wurden die Befragungsergebnisse mit den Erfahrungen und Sichtweisen aus Interviews ergänzt und kommentiert. Es wurden drei Gespräche mit insgesamt sechs VertreterInnen der IÖB-Ressorts BMK und BMDW, des Fördergebers/der Fördergeberin bzw. der Abwicklungsstelle aws und der IÖB-Servicestelle durchgeführt.

Da für das Modul Prepare gemäß Förderkonzept (noch) keine Lieferanten zur Verfügung standen wurden Gespräche zu Prepare sowie allgemein zu IÖB mit drei externen Expertinnen und Experten im Bereich innovationsfördernde öffentliche Beschaffung geführt. Darunter waren zwei Beraterlnnen, die die Gestaltung und Durchführung der IÖB-Challenges im Modul Prepare begleiteten.

\subsection{Stand und Ziele der Beschaffung}

Bei der gewünschten innovativen Lösung, die im Zuge des Moduls Transfer gefördert wurde, handelte es sich in $60 \%$ der Fälle um die Beschaffung eines Produkts. In vier Fällen (27\%) wurde sowohl ein Produkt als auch eine Dienstleistung (Beratungsleistung, Schulung) beschafft, in zwei Fällen nur eine Dienstleistung (13\%). Laut Angaben der öffentlichen AuftraggeberInnen befand sich die Beschaffung im Juli 2021 in der Mehrheit der Fälle noch am Anfang bzw. in der Umsetzung (vgl. Tab. 4). Ein Drittel ist seit kurzem, ein Viertel seit längerem abgeschlossen. Bei den LieferantInnen zeigte sich ein ähnliches Bild. Zu betonen ist, dass die Projekte, auf die sich die BeschafferInnen und LieferantInnen bei ihrer Beantwortung bezogen, nicht zwangsläufig dieselben waren.

Tabelle $4 \quad$ Umsetzungsstand Juli 2021

\begin{tabular}{|lcccc|}
\hline & \multicolumn{2}{c|}{ Beschafferlnnen } & \multicolumn{2}{c|}{ LieferantInnen } \\
\hline $\begin{array}{l}\text { Die Beschaffung / Lieferung der Lösung befindet sich erst am Anfang } \\
\text { bzw. in der Umsetzung }\end{array}$ & 6 & $40 \%$ & 2 & $40 \%$ \\
$\begin{array}{l}\text { Die Inbetriebnahme / Lieferung der Lösung ist seit kurzem abgeschlos- } \\
\text { sen (liegt weniger als 3 Monate zurück) }\end{array}$ & 5 & $33,3 \%$ & 2 & $40 \%$ \\
$\begin{array}{l}\text { Die Inbetriebnahme / Lieferung der Lösung ist seit längerem abgeschlos- } \\
\text { sen (liegt länger als 3 Monate zurück) }\end{array}$ & 4 & $26,7 \%$ & 1 & $20 \%$ \\
\hline & 15 & $100 \%$ & 5 & $100 \%$ \\
\hline
\end{tabular}

Im Falle von Modul Prepare wurden in einem Projekt zum Zeitpunkt der Befragung ausgewählte Einreichungen zum Innovationsdialog eingeladen. In einem anderen Fall war der Innovationsdialog bereits abgeschlossen, in einem weiteren Fall läuft eine Beauftragung im Nachgang der Challenge.

Transfer-FördernehmerInnen wurden insbesondere durch die Aktivitäten und Informationsarbeit der aws (40 \%) auf das Förderprogramm aufmerksam (vgl. Abb. 1). Eine wichtige Rolle nahmen auch private Unternehmens-, InnovationsberaterInnen oder ähnliche Einrichtungen (33 \%) ein. Zwei der befragten LieferantInnen im Modul Transfer gaben an, dass die Beschaffung bzw. der Auftrag an sie aufgrund früherer Kooperationen mit dem Beschaffer/der Beschafferin erfolgte. In zwei weiteren Fällen kam der Kontakt zwischen Beschafferln und Lieferantln über private BeraterInnen zustande, in einem anderen Fall über einen früheren Kooperationspartner des Lieferanten. 
Abbildung 1 Relevanz verschiedener Informationskanäle (Transfer)

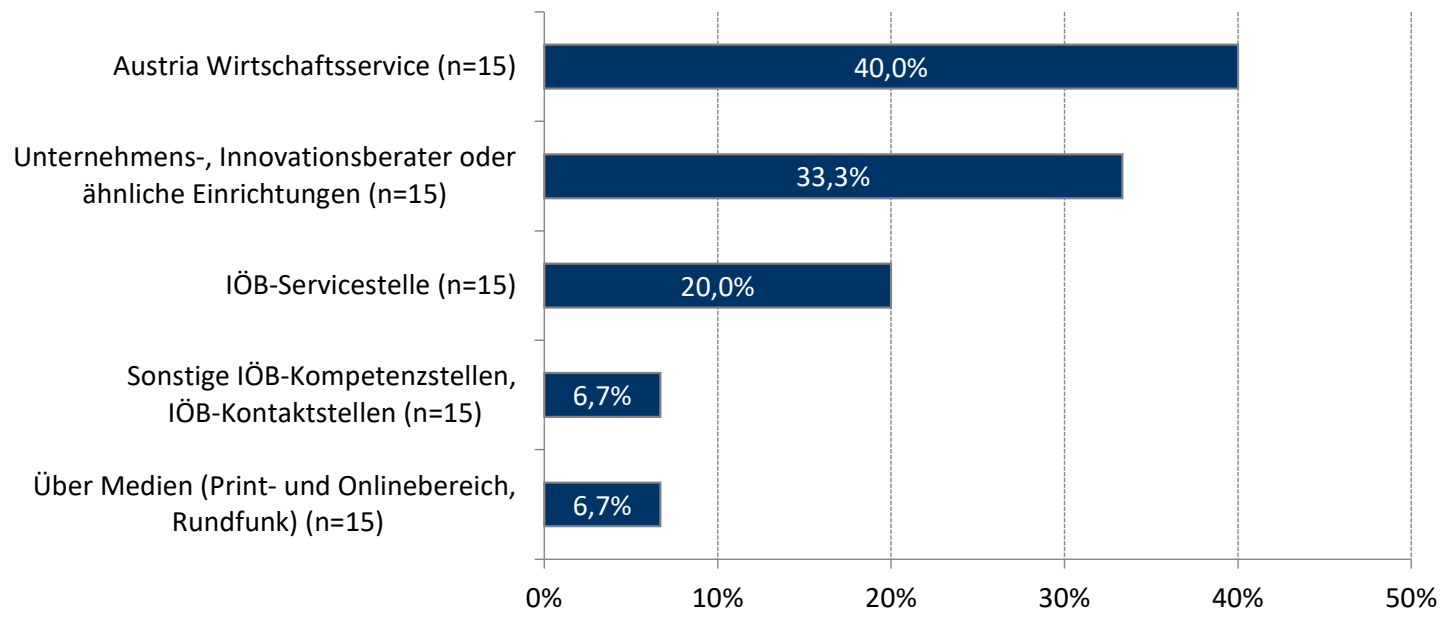

Quelle: Befragung öffentlicher BeschafferInnen im Modul Transfer. Mehrfachantworten möglich.

Die drei Prepare-FördernehmerInnen erfuhren von der IÖB-Toolbox über die aws, private BeraterInnen sowie die IÖB-Servicestelle. Die BeraterInnen von zwei der drei Prepare-Projekte gaben an, dass die Vorhaben durch bereits bestehende Kontakte in ihrem KundInnen-Netzwerk zustande kamen.

Gefragt nach den Gründen, warum sich die öffentlichen Beschafferlnnen im Modul Transfer um eine Förderung bemüht haben, hoben sieben von neun, die hierzu im Freitextfeld eine Angabe machten, das Angebot / die Nutzung / den Vorteil einer finanziellen Förderung / einer Fördermöglichkeit für innovative Beschaffung hervor. Jeweils einer der Befragten nannte die gute Vernetzung mit Unternehmen und die Möglichkeit, den öffentlichen Auftritt zu stärken, als Grund.

Aus der Befragung geht weiters hervor, dass sechs von 15 öffentlichen Beschafferlnnen (40\%) das Projekt auch ohne die Inanspruchnahme der Förderung unverändert umgesetzt hätten. Zwei $(13,3 \%)$ hätten das Vorhaben ohne die Förderung in reduziertem Umfang, zwei weitere $(13,3 \%)$ mit inhaltlichen Abstrichen durchgeführt. Die restlichen fünf der 15 Beschafferlnnen $(33,3 \%)$ hätten ohne die Förderung das Projekt bzw. die Beschaffung nicht in Angriff genommen.

Zu den Zielen, die mit der geförderten innovativen Lösung vom Beschaffer/von der Beschafferin verfolgt wurden bzw. werden, zählt in nahezu allen Fällen die Verbesserung / Erneuerung von Prozessen, Abläufen und Verfahren (vgl. Abb. 2). Die Verbesserung / Steigerung der Effizienz des Angebots bzw. der Services wurde etwas höher bewertet als die allgemeine Verbesserung / Erneuerung des Angebots, der Services bzw. des Zugangs dazu. Eine reine Verbesserung / Erneuerung der Gebäudeinfrastruktur und/oder der Raumsituation war nur in zwei Projekten Thema. Die LieferantInnen, von denen die geförderte Lösung beschafft wurde, nannten am häufigsten eine Verbesserung / Erneuerung von Prozessen, Abläufen und Verfahren und bestätigen damit das von den BeschafferInnen gezeichnete Bild. 
Abbildung 2 Ziele, die mit der Beschaffung verfolgt wurden bzw. werden (Transfer)

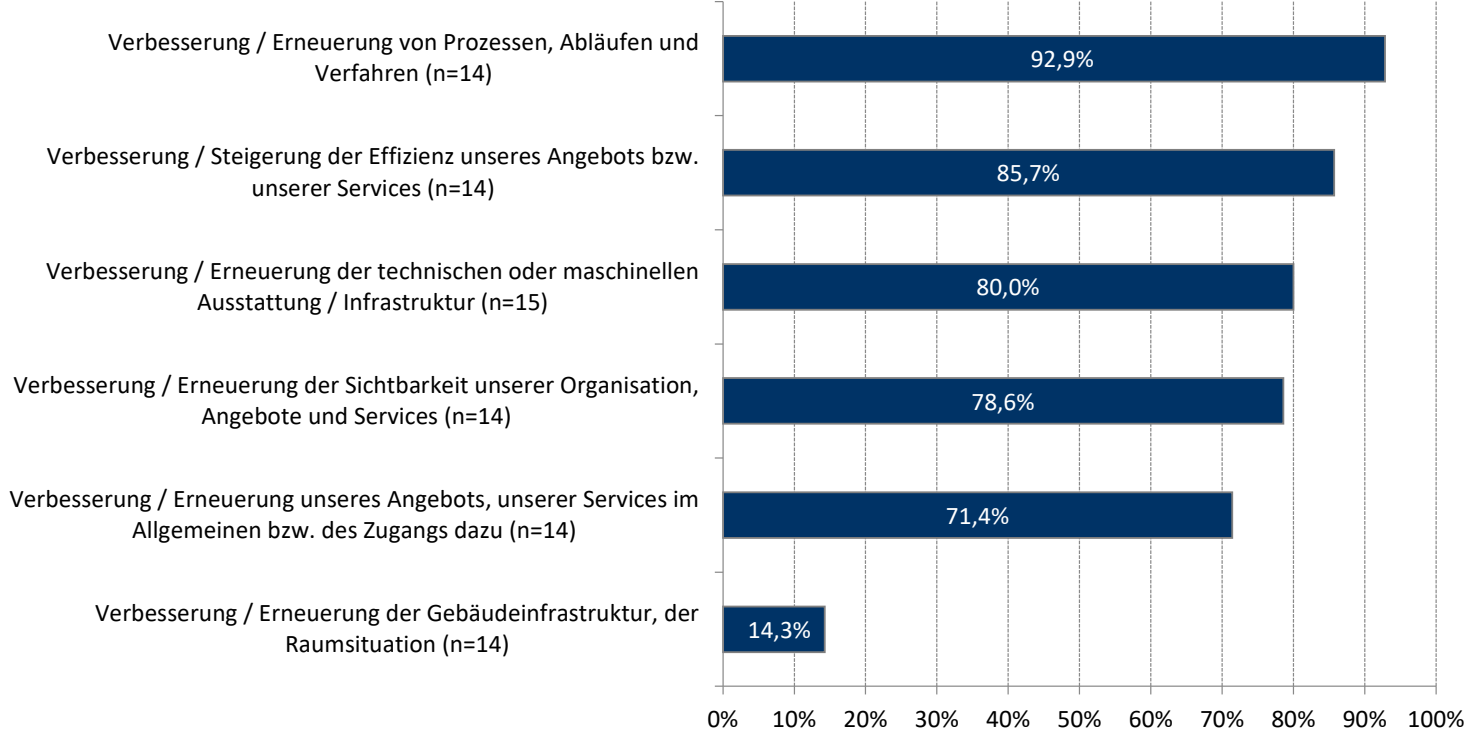

Quelle: Befragung öffentlicher BeschafferInnen im Modul Transfer. Rest auf 100 = Stimme nicht zu, nicht zutreffend.

Ziele der Prepare-Projekte ist die Planung und Umsetzung von IÖB-Challenges als Instrument der Markterkundung. Als öffentliche(r) Beschafferln die Möglichkeit zu erhalten, sich über innovative Lösungen zu informieren und „neue Ideen reinzubekommen“ schätzen die Beraterlnnen als sehr wertvoll ein. Demnach wurden den Prepare-Fördernehmerlnnen, also dem/der (potentiell künftigen) Beschafferln, einige Aspekte und Details erst im Zuge der Challenge klar: Einerseits durch den Austausch mit den Beraterlnnen in der Vorbereitung, andererseits durch die Vorschläge und Ideen der (potentiellen) LieferantInnen. Aus Sicht der BeraterInnen wurde dieser „Blick von außen“ von den Prepare-FördernehmerInnen positiv wahrgenommen. Ohne die Challenge, so vermuten die Beraterlnnen, wäre zwar eine Lösung beschafft worden, jedoch mit deutlich geringerem Innovationsgehalt.

Wie oben berichtet und in der Befragung bestätigt, kam es in einigen wenigen Transfer-Projekten (drei von 15) zu Abweichungen vom ursprünglichen Plan. In zwei Fällen wurde dies von den BeschafferInnen mit der Entwicklung der COVID-19-Pandemie begründet. Ein PrepareProjekt berichtete von (unerwarteten) Schwierigkeiten / einem höheren Aufwand bei der Vorbereitung bzw. Durchführung der IÖB-Challenge. Die Pandemie hatte laut externen Expertlnnen insofern ein Problem dargestellt, dass die Unternehmen sich nicht persönlich vor Ort ein Bild von der Herausforderung, die die IÖB-Challenge adressiert, machen konnten.

Im Zusammenhang mit dem geförderten Projekt bzw. der Beschaffung der innovativen Lösung wurden von den Transfer-Fördernehmerlnnen nur wenige Problembereiche benannt (vgl. Abb. 3). Knapp ein Drittel der Befragten berichtet von allgemeinen Schwierigkeiten / einem höheren Aufwand bei der Beschaffung der innovativen Lösung (21\% unerwartet, $13 \%$ erwartet) bzw. von Schwierigkeiten / einem höheren Aufwand bei der Integration der innovativen Lösungen (20\% unerwartet, $13 \%$ erwartet). Mangelndes Interesse der Organisation am Beschaffungsvorhaben konnte nur in einem Fall (unerwartet) attestiert werden. Interessant ist die Feststellung, dass die Erbringung der Eigenleistung für die Förderung nur in einem Fall (wie erwartet) ein Problem darstellte. 


\section{Abbildung 3 Probleme bei der geförderten Beschaffung (Transfer)}

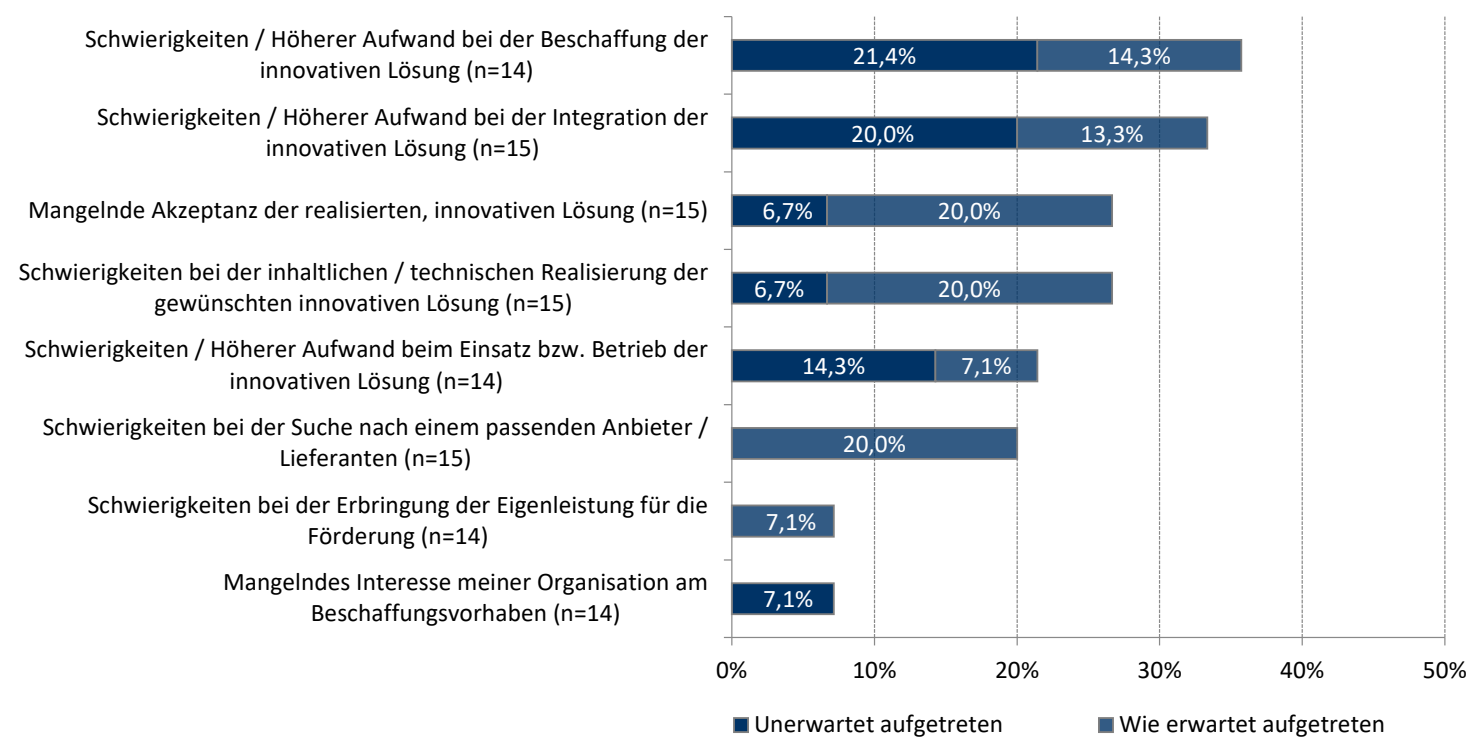

Quelle: Befragung öffentlicher BeschafferInnen im Modul Transfer. Rest auf 100: Nicht aufgetreten.

Zu den Maßnahmen, die sich laut Transfer-Fördernehmerlnnen positiv auf die Vermeidung / Bewältigung der benannten Problembereiche ausgewirkt haben, zählten laut Angabe in den Freitexten (Einzelnennungen) die intensive Vorab-Abklärung über Relevanz und Notwendigkeit der Beschaffung und (zeitgerechte) Abstimmung mit allen involvierten Bereichen, die Bündelung an Informationen, eine transparente Kommunikation sowie, in einem Fall, die Verlängerung der Projektlaufzeit (,hat den Druck reduziert“). Die Frage, ob die Förderung zu diesen Maßnahmen einen Beitrag geleistet hat, wurde von sieben Befragten im Freitextfeld mit "Ja“ beantwortet.

Zu den größten Herausforderungen, mit denen die LieferantInnen im Modul Transfer bei der Umsetzung des geförderten Projekts konfrontiert waren, zählten laut Freitextangabe (Einzelnennungen) die Schaffung eines gemeinsamen Verständnisses hinsichtlich der Aufgabenstellung und -teilung sowie der Berücksichtigung aller Interessen und Bedürfnisse der Stakeholder, die Konfrontation mit einem neuen Anwendungsgebiet, technische Problemstellungen, die Gewährleistung einer reibungslosen Umsetzung und das Aufsetzen und Ausformulieren des Angebots. Als Erfolgsfaktoren wurden die professionelle Vorgangsweise von allen Seiten, die kurzen Entscheidungswege, das Interesse am Thema, das Einbeziehen von Bedarfen potentieller Nutzerlnnen, das engagierte Projektteam und die gute Zusammenarbeit mit externen Partnerlnnen hervorgehoben (Einzelnennungen).

Laut Prepare-Fördernehmerlnnen hat die geförderte Beratung gut über die Anforderungen zum Aufruf einer Challenge informiert, bei der Ausgestaltung der Challenge als auch nach Abschluss der Challenge hochwertig und effizient unterstützt und wesentlich zu einer optimalen Durchführung der Challenge beigetragen (stimme sehr zu/stimme zu). 


\subsection{Ergebnisse und Wirkungen}

Viele der in Abb. 2 dargelegten Ziele wurden zum Zeitpunkt der Befragung bereits in großem Umfang erreicht. Jene öffentlichen BeschafferInnen, die sich das Ziel einer Verbesserung / Erneuerung von Prozessen, Abläufen und Verfahren gesetzt hatten, konnten dieses zu fast 50 \% (vollständig), 15,4 \% (zu einem großen Teil) bzw. 7,7 \% (teilweise) erreichen. Das Ziel einer Verbesserung / Erneuerung der technischen oder maschinellen Ausstattung / Infrastruktur wurde zu knapp 60 \% (vollständig) bzw. 17 \% (zu einem großen Teil) erreicht. Die zwei Projekte, die eine reine Verbesserung / Erneuerung der Gebäudeinfrastruktur und/oder der Raumsituation zum Ziel hatten, haben dieses bereits vollständig bzw. zu einem großen Teil erreicht. Die übrigen Ziele, die von den öffentlichen BeschafferInnen benannt wurden, konnten zu insgesamt ca. 70 \% (Summe von vollständig, zu einem großen Teil, teilweise) erreicht werden. Zu berücksichtigen gilt an dieser Stelle wieder, dass sich ein Teil der Fälle noch am Anfang bzw. in der Umsetzung befindet und einige Ziele noch gar nicht erreicht werden konnten.

Wie eine rezente Untersuchung ${ }^{11}$ für Österreich zeigt, können innovative öffentliche Beschaffungsvorhaben ein breites Wirkungsspektrum entfalten. Wirkungen können sich in unterschiedlichen Dimensionen einstellen, wobei deren Realisation von zahlreichen kontextuellen Bedingungen abhängt. Bei spezialisierten Projekten ist der Wirkungskorridor schmäler. Auch in der gegenständlichen Untersuchung zeigen sich verschiedene Wirkungsweisen der geförderten Projekte, die fachlich und inhaltlich unterschiedlich ausgestaltet sind. Ziel der nachfolgenden Betrachtung ist die Darstellung eines Wirkungsprofils aus der Perspektive der Ziele des Förderprogramms und nicht eine Wirkungsbewertung auf der Ebene einzelner Projekte.

Im Hinblick auf die Ziele, einen Beitrag zur IÖB-Initiative zu leisten und die Sichtbarkeit von IÖB zu erhöhen, ist die Vorbildwirkung für andere öffentliche Verwaltungsbereiche oder Einrichtungen hervorzuheben (vgl. Abb. 4). Laut Einschätzungen von öffentlichen Beschafferlnnen im Modul Transfer haben etwa $36 \%$ der Vorhaben bereits in kurzer Zeit zu einer Vorbildwirkung beigetragen, die Hälfte erwartet eine entsprechende Entwicklungen mittel bis langfristig. Ferner konnten aus Sicht der Befragten in einem Drittel der Projekte bereits eine Steigerung der Effizienz und/oder Effektivität des eigenen Angebots bzw. der eigenen Services erreicht werden (13\% kurzfristig, $33 \%$ langfristig erwartet). Mehr als ein Viertel der Projekte hat dazu beigetragen, die Professionalität des Angebots bzw. der Services zu steigern (13 \% kurzfristig, $40 \%$ langfristig erwartet). Die Erwartung, dass durch die Vorhaben künftig weitere Beiträge zur Verbesserung der Angebote und Services geleistet werden, ist hoch. Besonders interessant: Drei Projekte geben an, eine öffentliche Auszeichnung oder einen Preis im Kontext der Beschaffung erhalten zu haben.

\footnotetext{
${ }^{11}$ Ploder, M., Streicher, J., Buchinger, E. und Kienegger, M. (2019): IÖB Wirkungsanalyse. Tiefergehende, wirkungsorientierte Analyse von innovationsfördernden öffentlichen Beschaffungsprojekten in unterschiedlichen Anwendungsfeldern. Projektbericht Joanneum Research und Austrian Institute of Technology, Wien und Graz.
} 


\section{Abbildung 4 Wirkungen mit Fokus auf den öffentlichen Beschaffer (Transfer)}

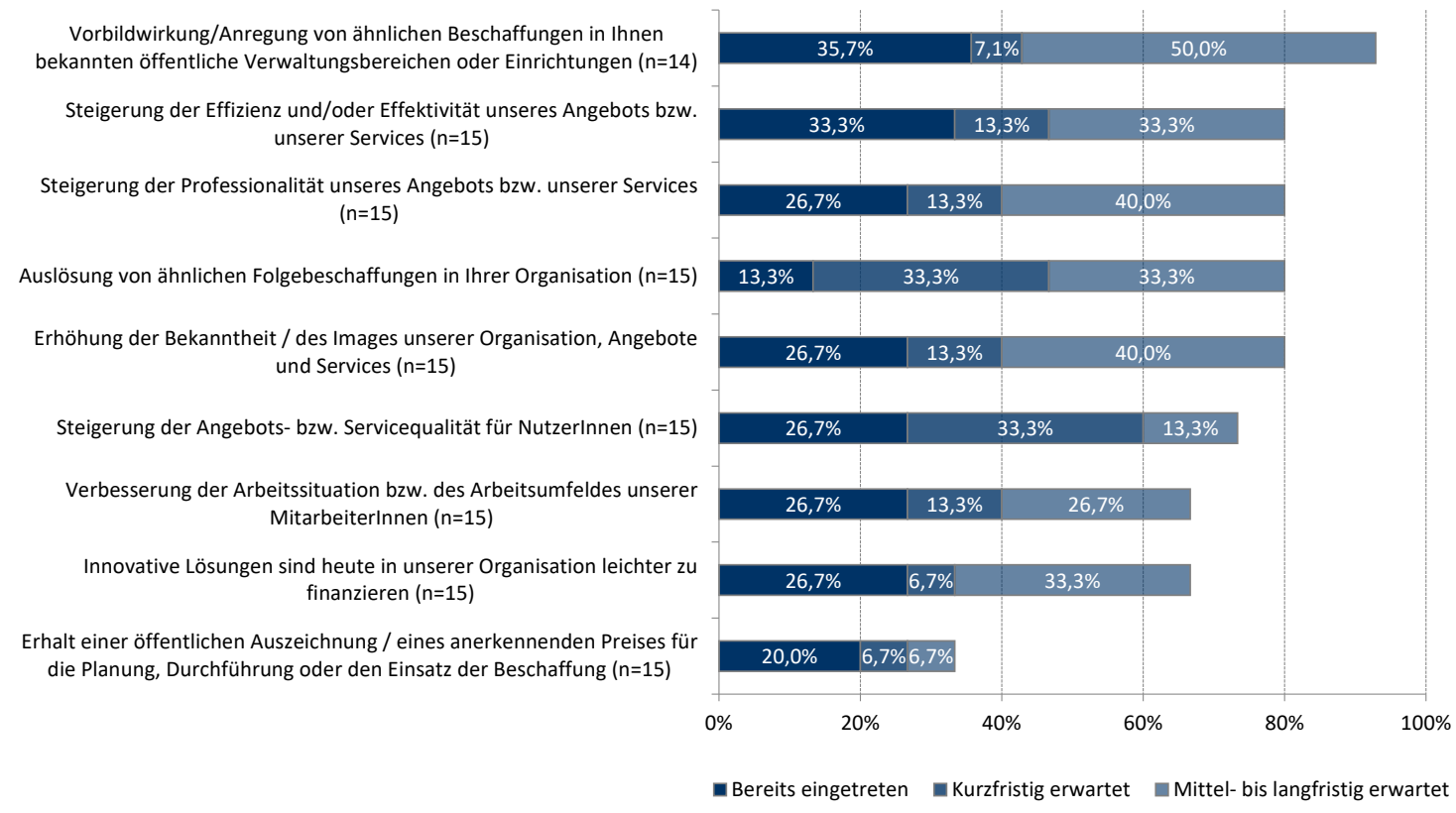

Quelle: Befragung öffentlicher BeschafferInnen im Modul Transfer. Rest auf 100: Nicht zu erwarten bzw. nicht zutreffend.

Im Falle der drei Prepare-FördernehmerInnen konnte, neben der Identifikation / Auswahl einer innovativen Lösung für die Challenge und der Identifikation / Auswahl eines/einer innovativen Lieferanten/Lieferantin (bereits eingetreten oder kurzfristig erwartet), mit einem Projekt bereits eine Vorbildwirkung erzielt werden (dazu zwei mittel-/langfristig erwartet). Ein weiteres trug dazu bei, die Sensibilität und das Verständnis für die Beschaffung innovativer Lösungen in der Organisation zu erhöhen (dazu einer mittel-/langfristig erwartet).

Aus Sicht der Fördernehmerlnnen im Modul Transfer haben $40 \%$ der Vorhaben bereits zu einer Steigerung der Effizienz der öffentlichen Hand beigetragen (vgl. Abb. 5). In weiteren $40 \%$ sind entsprechende Steigerungen in naher bzw. mittel- bis langfristiger Zukunft zu erwarten. Während laut den Befragten 20 \% der Vorhaben bereits dazu beigetragen haben, die Innovationskraft der heimischen Wirtschaft zu stärken, soll dieser Beitrag mittel- bis langfristig noch deutlich steigen (66 \%). Ein Drittel der Projekte leistete einen Beitrag zur Nachhaltigkeit und Umweltverträglichkeit. Auch hier sind die Erwartungen an künftige Entwicklungen hoch (insg. 40\%). Zwar fanden sich weniger Projekte mit (erreichten, erwarteten) Beiträgen zu Gesundheit und Wohlbefinden bzw. Chancengleichheit und -gerechtigkeit, was primär auf die Inhalte und Ausgestaltung der einzelnen Projekte zurückzuführen ist. Andererseits ist es ein Beleg für die verschiedenen Wirkungsweisen von IÖB und somit durchaus als Erfolg zu werten. 


\section{Abbildung 5 Wirkungen mit Fokus auf den gesellschaftlichen Mehrwert (Transfer)}

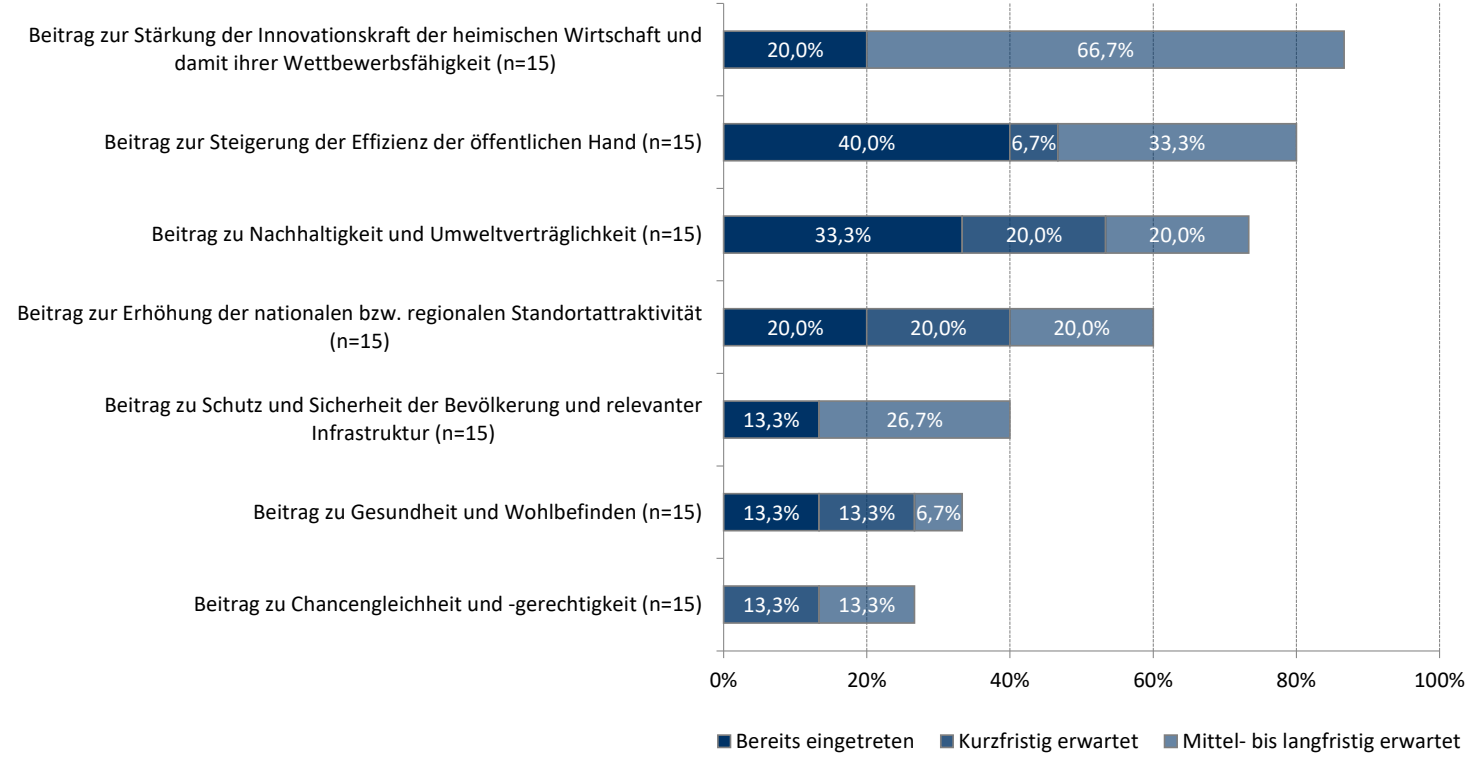

Quelle: Befragung öffentlicher BeschafferInnen im Modul Transfer. Rest auf 100: Nicht zu erwarten bzw. nicht zutreffend.

Jeweils drei von fünf der befragten LieferantInnen im Modul Transfer gaben an, dass die Innovationsnachfrage aus dem öffentlichen Sektor zu einer positiven Umsatzentwicklung von Produkten / Dienstleistungen beigetragen (dazu einer kurzfristig, einer mittel-/langfristig erwartet), eine Verbesserung von Kompetenzbereichen (z.B. F\&E, Innovation) mit sich gebracht (dazu einer kurzfristig erwartet) und zu einer erhöhten Sensibilität und Verständnis für F\&E sowie Innovation im Unternehmen (dazu einer mittel-/langfristig erwartet) geführt hat. Jeweils zwei von fünf Unternehmen nutzen das Projekt bereits als Referenz (dazu zwei mittel-/langfristig erwartet), für die Präsentation auf Veranstaltungen (dazu zwei kurzfristig, einer mittel-/langfristig erwartet) und für die Erschließung neuer Innovations- bzw. Geschäftsfelder (dazu einer kurzfristig, einer mittel-/langfristig erwartet). Vergleichsweise weniger Einfluss hatte das Projekt auf die Verbesserung von (eigenen) betrieblichen Abläufen und Prozessen und die Anmeldung von gewerblichen Schutzrechten.

In den Sonderrichtlinien von 2021 wurden erstmals Wirkungsindikatoren zur Beurteilung der Zielerreichung des Programms eingeführt (vgl. Tab. 5). Ergänzend zur Erfassung des Wirkungsspektrums wurden diese in der gegenständlichen Evaluation herangezogen um eine gesamthafte Abschätzung der Entwicklung der 2019/2020 geförderten Projekte vorzunehmen. Hierfür wurden die öffentlichen BeschafferInnen im Modul Transfer und deren LieferantInnen in der Online-Erhebung gefragt, wie sich die Beschaffung bzw. der Einsatz der innovativen Lösung insgesamt ausgewirkt hat bzw. auswirken wird. Aufgrund des Förderkonzepts wurden die Wirkungsindikatoren nicht auf das Modul Prepare angewendet. 
Tabelle $5 \quad$ Ziele und Wirkungsindikatoren (Impact-Dimension)

\begin{tabular}{|c|c|}
\hline Ziel & Indikator, Zielwert und Frage in der Online-Erhebung \\
\hline $\begin{array}{l}\text { Positive Wirkungen durch Be- } \\
\text { schaffung und Einsatz von Inno- } \\
\text { vationen im öffentlichen Sektor }\end{array}$ & $\begin{array}{l}\text { Von den geförderten öffentlichen Auftraggebern wahrgenommene positive Auswir- } \\
\text { kungen (Zielwert: }>75 \% \text { der Förderungsnehmer bestätigen positive Auswirkungen } \\
\text { in Bezug auf deren Aufgaben, wie beispielsweise Effizienzsteigerung, Kostenein- } \\
\text { sparung, Reduktion negativer Umwelt- und Klimawirkungen, verbesserte Services). }\end{array}$ \\
\hline Frage in der Online-Erhebung & $\begin{array}{l}\text { Zusammenfassende Einschätzung: Wurde bzw. wird durch die Beschaffung / durch } \\
\text { den Einsatz der innovativen Lösung eine Verbesserung im Vergleich zur Aus- } \\
\text { gangssituation erzielt bzw. wird dies erwartet (z.B. Effizienzsteigerung, Kostenein- } \\
\text { sparung, Reduktion negativer Umwelt- und Klimawirkungen, verbesserte Services, } \\
\text { etc.)? Antwortmöglichkeiten: Wesentlich verbessert / etwas verbessert / bleibt un- } \\
\text { gefähr gleich / eventuell verschlechtert. }\end{array}$ \\
\hline $\begin{array}{l}\text { Positive Wirkungen auf österrei- } \\
\text { chische Unternehmen (als Anbie- } \\
\text { ter/Lieferanten von Innovationen) } \\
\text { durch die Innovationsnachfrage } \\
\text { aus dem öffentlichen Sektor }\end{array}$ & $\begin{array}{l}\text { Von den Lieferanten in Österreich wahrgenommene positive Auswirkungen (Ziel- } \\
\text { wert: }>50 \% \text { der Lieferanten bestätigen positive Auswirkungen in Bezug auf die Wir- } \\
\text { kung des öff. Sektors als Referenzkunde und in Bezug auf aktuelle/zukünftige Inno- } \\
\text { vationsaktivitäten, wie bspw. positive Umsatzentwicklung, erhöhte Sichtbarkeit als } \\
\text { Lieferant für den öffentlichen Sektor, Anreize für zukünftige Innovationsaktivitäten). }\end{array}$ \\
\hline Frage in der Online-Erhebung & $\begin{array}{l}\text { Zusammenfassende Einschätzung: Wurde bzw. wird durch die Innovationsnach- } \\
\text { frage aus dem öffentlichen Sektor eine Verbesserung der Aktivitäten und Entwick- } \\
\text { lungen in Ihrem Unternehmen erzielt bzw. wird dies erwartet (z.B. positive Umsatz- } \\
\text { entwicklung, erhöhte Sichtbarkeit als Lieferant für den öffentlichen Sektor, Anreize } \\
\text { für zukünftige Innovationsaktivitäten, etc.)? Antwortmöglichkeiten: Wesentlich ver- } \\
\text { bessert / etwas verbessert / bleibt ungefähr gleich / eventuell verschlechtert. }\end{array}$ \\
\hline
\end{tabular}

Quelle: IÖB-Toolbox. Sonderrichtlinien 2021. Befragung öffentlicher Beschafferlnnen im Modul Transfer und deren LieferantInnen.

Nahezu alle öffentlichen BeschafferInnen im Modul Transfer erzielten bzw. erwarten wesentliche Verbesserungen durch die Beschaffung / durch den Einsatz der innovativen Lösung im Vergleich zur Ausgangssituation (vgl. Abb. 6). Bei drei der fünf LieferantInnen wurde bzw. wird durch die Innovationsnachfrage aus dem öffentlichen Sektor eine wesentliche Verbesserung der Aktivitäten und Entwicklungen in ihrem Unternehmen erzielt bzw. wird hier erwartet. Die restlichen zwei erzielten/erwarten etwas Verbesserung durch das Projekt. Damit werden die in den Sonderrichtlinien anvisierten Zielwerte (75\% der Beschafferlnnen bzw. 50 \% der LieferantInnen bestätigen positive Auswirkungen) bereits in der ersten Programmphase erreicht bzw. übertroffen. Einschränkend ist bei diesen Ergebnissen darauf hinzuweisen, dass in der OnlineErhebung aufgrund der Vielzahl an laufenden Projekten auch Erwartungen abgefragt wurden. Zudem wurde mit der Befragung nur eine kleine Anzahl an LieferantInnen erreicht. 
Abbildung 6 Verbesserung durch die Beschaffung im Vergleich zur Ausgangssituation
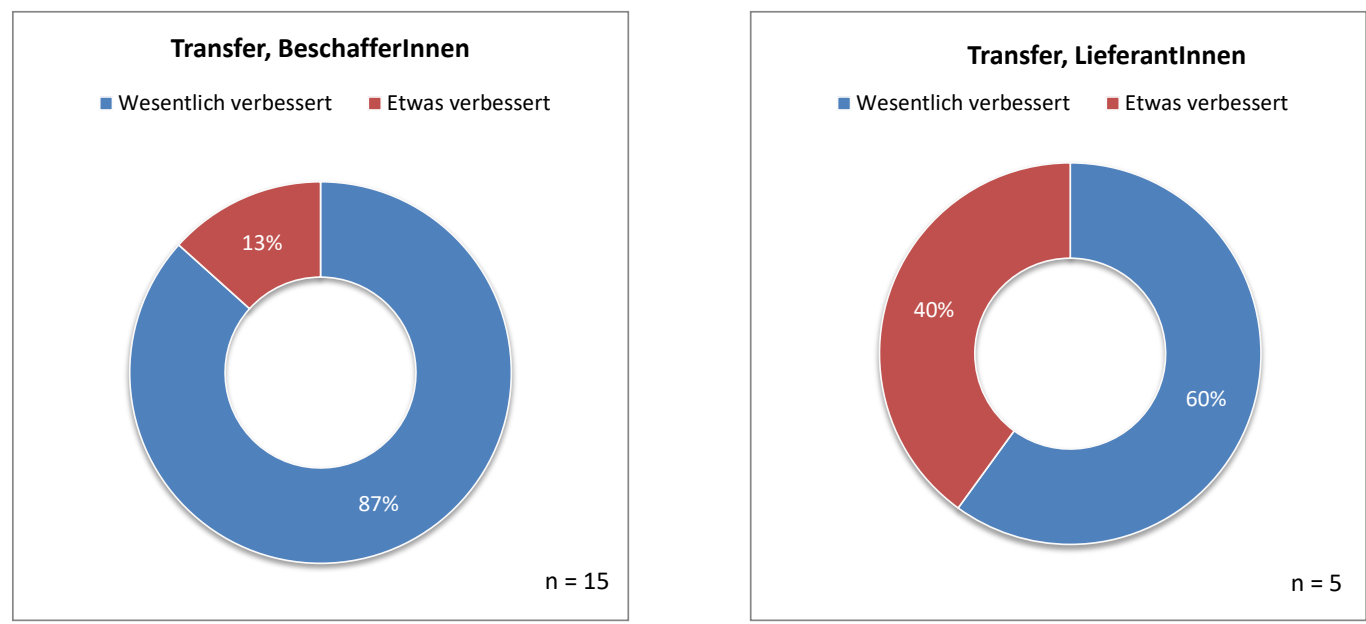

Quelle: Befragung öffentlicher BeschafferInnen und LieferantInnen im Modul Transfer. Weitere Antwortmöglichkeiten: „Bleibt ungefähr gleich“, „Eventuell verschlechtert“.

\subsection{Wahrnehmung des Förderverfahrens}

Im Zuge der Befragung der öffentlichen Beschafferlnnen im Modul Transfer wurde auch um eine Beurteilung unterschiedlicher Aspekte des Förderverfahrens gebeten (vgl. Abb. 7). Dabei wurde die technisch-operative Hilfestellung durch die aws sowie die bereitgestellten Informationen zur Förderung und zum Förderverfahren jeweils von 67 \% der öffentlichen Beschafferlnnen als sehr gut, von $27 \%$ als eher zufrieden eingestuft. Der administrative / bürokratische Aufwand im Vergleich zum gewonnen Nutzen durch die Förderung erhielt ähnlich hohe Zufriedenheitswerte. Vergleichsweise weniger zufrieden waren die Befragten mit der Dauer von der Projekteinreichung bei der aws bis zum Erhalt des Vertrages.

Mangels direkten Austauschs konnten die LieferantInnen im Modul Transfer keine Auskunft zum Förderverfahren geben. Fördernehmerlnnen im Modul Prepare schätzen die genannten Aspekte des Förderverfahrens und der Förderabwicklung im Schnitt etwas weniger positiv ein. 


\section{Abbildung 7 Einschätzung des Förderverfahrens und der-abwicklung (Transfer)}

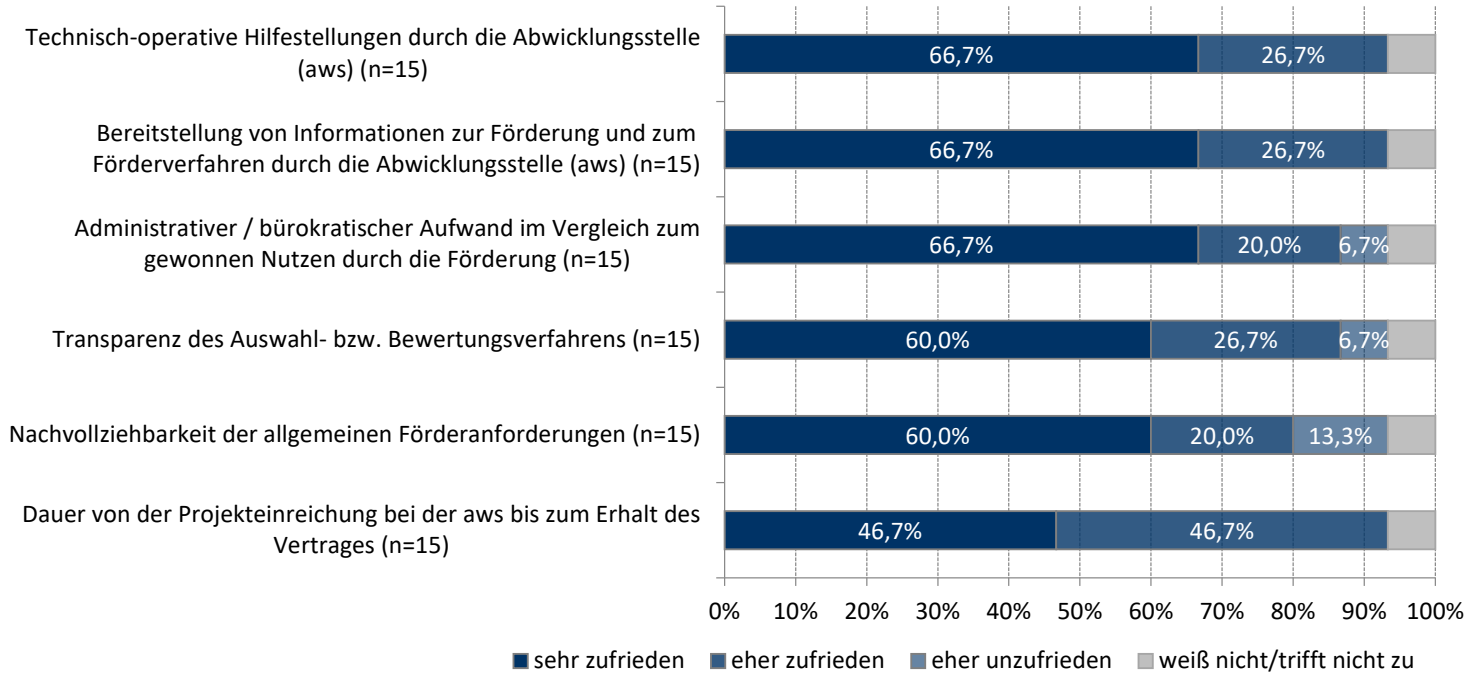

Quelle: Befragung öffentlicher BeschafferInnen im Modul Transfer.

Ein höherer Zuschuss im Rahmen der Förderung hätte im Falle von Transfer nur für $29 \%$ etwas am Projekt bzw. an der Beschaffung geändert (Prepare: einer von drei). Laut Freitextnennungen hätten dadurch technische Details (noch) besser adressiert und ergänzende Maßnahmen umgesetzt werden können. Der Durchführungszeitraum, in dem das Projekt umgesetzt werden soll, war für drei Viertel der Befragten angemessen (Prepare: zwei von drei).

\subsection{Verbesserungspotentiale aus Sicht der Befragten}

Während zwei öffentliche Beschafferlnnen in den Freitextnennungen aktuell keine Verbesserungsbedarfe erkennen können, sehen drei andere Beschafferlnnen Optimierungspotentiale hinsichtlich der Vereinfachung und Beschleunigung des Antragsverfahrens. Dabei wird insbesondere auf die auf kommunaler Ebene oftmals geringen Ressourcen für die Bearbeitung und Einreichung solcher Anträge hingewiesen.

In den Interviews mit BeraterInnen von Prepare-Projekten wurde die mangelnde Bekanntheit und Sichtbarkeit von Prepare thematisiert. Gute Erfahrungen wurden mit der direkten Ansprache von (potentiellen) Beschafferlnnen gemacht. Aus Sicht der Beraterlnnen könnte die Bekanntheit von Prepare bzw. der IÖB-Toolbox durch verstärkte Bewerbung auf gemeinsamen Veranstaltungen von Städten und Gemeinden gesteigert werden. Andererseits sollte der gezielte Austausch von öffentlichen BeschafferInnen über Erfolge und Herausforderungen der Beschaffung forciert werden. Mit Blick auf Prepare wäre zu prüfen, ob sich mehrere öffentliche Beschafferlnnen mit ähnlichen Herausforderungen für die Gestaltung und Durchführung einer IÖB-Challenge finden würden. 


\section{Zusammenfassung und Schlussfolgerungen}

Die IÖB-Toolbox wurde 2019 als neues Format mit dem Ziel gestartet, öffentlichen Auftraggeberlnnen die Durchführung von innovativen Beschaffungen (Modul Transfer) sowie die Planung und Umsetzung von IÖB-Challenges (Modul Prepare) zu ermöglichen. Während sich das Modul Transfer an öffentliche BeschafferInnen mit einem konkreten Bedarf an einem innovativen Produkt oder an einer Dienstleistung richtet, soll mithilfe des Moduls Prepare die Recherche nach innovativen Ideen und Lösungsvorschlägen unterstützt und die Chance, dass innovative Produkte oder Dienstleistung im öffentlichen Bereich eingesetzt werden, erhöht werden. Ähnlich wie der Vorgänger, IÖB-Projektwettbewerb, soll die Förderung der Beschaffung bzw. der Beratungsleistung im Rahmen der IÖB-Toolbox konkret dazu beitragen, die Sichtbarkeit von IÖB im Allgemeinen zu erhöhen, die Anzahl und Qualität innovativer Beschaffungsprojekte zu steigern und daraus „Vorzeigeprojekte“ bzw. "Good-Practices“ entstehen zu lassen. Von der Beschaffung und dem Einsatz der innovativen Lösung werden positive Wirkungen im öffentlichen Sektor (nachfrageseitig) sowie auf österreichische Unternehmen (angebotsseitig) erwartet.

Die gegenständliche Evaluation adressiert die Umsetzung des Programms IÖB-Toolbox in der ersten Programmphase (2019-2020), der bis dato feststellbaren Beiträge zu Zielerreichung und erster Wirkungen. Eine Bewertung der Qualität der innovativen Beschaffungsprojekte war nicht Gegenstand dieser Untersuchung. Hauptaugenmerk galt der Konzeption und Durchführung einer Befragung der FörderungsnehmerInnen (öffentliche Beschafferlnnen) sowie deren LieferantInnen im konkreten Projekt. Für die Untersuchung relevante Daten und Dokumente wurden von der aws zur Verfügung gestellt.

\section{Programmumsetzung 2019-2020}

Die IÖB-Toolbox fügt sich gut in das bestehende IÖB-Instrumentarium ein. Im Innovationsförderkontext grenzt sich das Programm als beim Beschaffer (nachfrageseitig) ansetzendes Instrument mit hohem Innovationsfokus und attraktivem finanziellen Anreiz ab. Das Programm wird durch eine unternehmerisch orientierte, in der Beurteilung von Innovationen erfahrene, langjährige IÖB-Kompetenzstelle betreut und abgewickelt. Die Zusammenarbeit und Abstimmung zwischen den relevanten Akteuren wird als eng und produktiv beschrieben. Neben Projekten mit hohem Bezug zu „grünen Themen“ wie Umwelt- und Klimaschutz oder Nachhaltigkeit war Digitalisierung dominierendes Thema in den eingegangenen Anträgen.

Die Programmdokumente der ersten Phase (2019-2020) skizzieren die Bedarfslagen und Herausforderungen innovationsfördernder öffentlicher Beschaffung in entsprechender Weise. Die Förderkriterien sind klar ausgewiesen. Die zur Bewertung der Projekte zur Anwendung kommenden inhaltlichen Kriterien sind ebenfalls gut nachvollziehbar, spiegeln aber nicht immer die Zielsetzungen der Förderung in angemessener Art und Weise wider. Output-Indikatoren werden gelistet, es fehlen jedoch Zielwerte und differenzierte, umsetzungsorientiere Zielsetzungen. Das Monitoring, das laut den Programmdokumenten zum Zwecke der Überprüfung der Wirkungen der Förderung eingerichtet werden soll, informiert nur über Aktivitäten und Outputs. Der Beitrag des Monitorings zur Programmumsetzung ist unklar.

Die in der ersten Phase geförderten Projekte befinden sich Mitte 2021 mehrheitlich am Anfang bzw. in der Umsetzung. Gänzlich abgeschlossen und mit der Förderstelle abgerechnet ist nur eine kleine Anzahl. Auf die IÖB-Toolbox aufmerksam wurden die öffentlichen Beschafferlnnen 
neben der Informationsarbeit der aws vor allem über private BeraterInnen. Zuletzt fanden einige über Empfehlungen („Mundpropaganda“) anderer öffentlicher AuftraggeberInnen zum Förderprogramm. Das auf Transfer in Summe mehr Projekte entfielen als auf Prepare lässt sich einerseits auf die Attraktivität einer finanziellen Förderung zurückführen, was in der Befragung auch mehrfach als Grund für die Teilnahme angeführt wurde. Zudem dürfte die COVID19-Pandemie einige der Projekte angestoßen und das Förderprogramm zusätzlich ins Blickfeld öffentlicher Beschaffer gerückt haben. Andererseits mussten einige Anträge zurückgezogen werden, da die Finanzierung aus Ressourcenmangel (doch) nicht sichergestellt werden konnte. Positiv hervorzuheben ist, dass viele neue, im Kontext von IÖB bisher weniger aktive Beschafferlnnen aus dem kommunalen Bereich zum Programm gefunden haben.

Im Gegensatz dazu wurde im Modul Prepare zu Beginn die Umsetzung von drei Challenges anvisiert. Das Förderkonzept sieht den Einsatz von externen BeraterInnen vor, die identifiziert, angesprochen und geschult („IÖB-Zertifikat“) werden mussten. Die externen Beraterlnnen informierten ihr Kundlnnen-Netzwerk hinsichtlich der Möglichkeiten und Anforderungen des Förderprogramms und machten sich aktiv auf die Suche nach passenden Projektideen. Bis interessante Projektideen schließlich gefunden und konzipiert werden konnten, verging jedoch etwas Zeit. Laut Prepare-Fördernehmerlnnen hat die geförderte Beratung den Weg zur IÖBChallenge und dessen Durchführung überaus gut unterstützt.

\section{Zielerreichung und Wirkungsweisen}

Viele der Ziele, die mit der geförderten innovativen Lösung verfolgt wurden bzw. werden, darunter die Verbesserung / Erneuerung von Prozessen, Abläufen und Verfahren oder die Verbesserung / Erneuerung der technischen oder maschinellen Ausstattung / Infrastruktur, wurden zum Zeitpunkt der Befragung bereits im großen Umfang erreicht. Weniger überraschend wurden jene Ziele, die vorwiegend technischer, operativer Natur sind, rascher erreicht, also jene, die allgemein länger für eine Entfaltung brauchen (z.B. Erhöhung der Sichtbarkeit der Organisation). Zu berücksichtigen gilt der frühe Umsetzungsstatus einiger Projekte. Insgesamt zeigen sich geringe Planabweichungen im Zuge der Beschaffung. Zudem werden seitens der Fördernehmerlnnen nur in wenigen Fällen Schwierigkeiten und Herausforderungen wahrgenommen, etwa bezüglich eines höheren Aufwandes bei der Beschaffung. Das Förderverfahren und die Förderabwicklung erhalten hohe Zufriedenheitswerte, insbesondere wird das Aufwand-/Nutzenverhältnis der Förderung als sehr positiv eingeschätzt.

Nach Einschätzung der öffentlichen BeschafferInnen konnten die Projekte bereits eine Reihe von Wirkungen entfalten, die in naher Zukunft zunehmend an Umfang gewinnen sollen. Hervorzuheben sind die Beiträge zu Schaffung einer Vorbildwirkung sowie zur Verbesserung des Angebots und der Services bei der/dem Beschafferln. Die Projekte tragen zu einer Steigerung der Effizienz der öffentlichen Hand bei, leisten einen Beitrag zu Nachhaltigkeit und Umweltverträglichkeit und stärken die Innovationskraft der heimischen Wirtschaft. Angebotsseitig, bei den LieferantInnen, werden eine positive Umsatzentwicklung, Verbesserungen in relevanten Kompetenzbereichen und eine erhöhte Sensibilität und erhöhtes Verständnis für F\&E sowie Innovation im Unternehmen wahrgenommen. Insgesamt erzielten bzw. erwarten alle öffentlichen BeschafferInnen im Modul Transfer und deren LieferantInnen wesentliche bzw. zumindest etwas Verbesserung(en) im Vergleich zur Situation vor der Beschaffung. Die in den Sonderrichtlinien von 2021 anvisierten Zielwerte werden dadurch erreicht bzw. übertroffen. 


\section{Fazit und Handlungsoptionen}

Die IÖB-Toolbox hat als neues Format zur Stärkung der IÖB beigetragen, auch durch die Mobilisierung wesentlicher Zielgruppen. Von der Förderung gehen wichtige Impulse für die IÖB aus. Im Falle von Prepare wird durch das BeraterInnen-Netzwerk die Arbeit und das Wirken der IÖB-Servicestelle stärker regionalisiert. Die Anzahl innovativer Beschaffungsprojekte wurde insgesamt gesteigert. Viele der Beschaffungsziele wurden bereits vollständig oder zumindest teilweise erreicht. Die Projekte entfalten Vorbildwirkung und schaffen Erfahrungen und Kenntnisse, von denen andere öffentliche Beschafferlnnen profitieren können. Einige Wirkungen konnten rasch erreicht werden, einige benötigen eine längere Anlaufzeit. Insgesamt erzielten bzw. erwarten sowohl Beschafferlnnen als auch LieferantInnen im Modul Transfer durch das Projekt teils deutliche Verbesserungen im eigenen Umfeld.

Während die Evaluierung die intendierten Wirkungsweisen bestätigt, die sich nach Realisierung der Projekte bereits eingestellt haben oder die erwartet werden, stellt sich die Frage, wie entscheidend die Förderung für die Umsetzung der Projekte war. Der Anteil der Beschafferlnnen, die laut eigenen Angaben die Projekte auch ohne Förderung umgesetzt hätten, fällt relativ hoch aus. Einerseits gilt zur berücksichtigen, dass Gemeinden und Kommunen größere Beschaffung zumeist lange im Voraus planen, hierfür Beschlüsse fassen und Vorfinanzierungen aufstellen müssen. Selbst bei einem negativ beschiedenen Förderantrag wird typischerweise entsprechend der Beschlusslage gehandelt, d.h. das Projekt dennoch umgesetzt. Andererseits hängt das Ausmaß der Mitnahme stark von der Zielgruppe der Förderung ab. Für kleine Gemeinden und Kommunen mit wenig Ressourcen schaffen Förderungen wie Transfer überhaupt erst die Möglichkeit, Innovationsbeschaffungen durchzuführen.

Mit Blick auf die Additionalität bedarf es daher weiter an Evidenz, auch mit Blick auf die Rolle der COVID-19-Pandemie. Im Rahmen künftiger Untersuchungen sollte geprüft werden, ob die unveränderte oder spätere Durchführung ohne Förderung tatsächlich den Innovationsgehalt erreicht hätte, der mit dem geförderten Projekt anvisiert worden war. Detaillierte Informationen dazu, was die Förderung warum angestoßen hat, werden als wichtig erachtet. In diesem Lichte sind auch die genaueren Beweggründe, die zum Verzicht auf die zugesagte Förderung bzw. die zur Beendigung des geplanten Projekts geführt haben, zu erheben und zu analysieren.

Wie bereits vergangene Studien zeigen konnten, ist bei IÖB ein „Promotor“ wichtig, dem die Innovationsthematik wichtig ist, der die Beschaffung vorantreibt und das dafür notwendige Management übernimmt. Auch in dieser Untersuchung wurde an einigen Stellen auf die Wichtigkeit einer guten Vorbereitung und Planung für ein erfolgreiches IÖB-Projekt hingewiesen. Die Möglichkeiten der Unterstützung von Beschaffungen durch die Toolbox, in welchem der Bedarf und/oder das Interesse einer Einrichtung mit dem mindestens einer anderen gebündelt wird, und wo sich Verantwortlichkeiten und Aufgaben teilen lassen, sollte geprüft werden. Prepare soll jedoch weiterhin nicht Bedingung für Transfer sein.

Die Rolle der externen Beraterlnnen ist als hoch und wesentlich für die Sichtbarkeit und Wahrnehmung des Förderprogramms und damit auch dessen Erfolgs einzuschätzen. Dieses Netzwerk wäre weiter auszubauen. Innerhalb dieses Netzwerkes könnte der Austausch auf Basis kleinerer Veranstaltungen („Meet-and-greet) forciert werden, bei welchen externe BeraterInnen über ihre Erfahrungen mit IÖB berichten. Um LieferantInnen stärker in ein Monitoring einzubin- 
den sind entsprechende Anreize erforderlich. Eine Möglichkeit wäre entsprechende Informationen und Daten über die Erfassung von „Success Stories“ einzuholen. Generell ist zu empfehlen, dass über Projekte und Erfahrungen mehr und in anschaulicherer Form berichtet wird.

Seit 2021 wird die IÖB-Toolbox im Rahmen der IÖB-Initiative aus Mitteln des BMK dotiert und weitergeführt. Soweit ersichtlich wurden einige der hier skizzierten Befunde in den Sonderrichtlinien von 2021 bereits aufgegriffen und Anpassungen vorgenommen, wie etwa eine klarere Zielsetzung mit Fokus auf Klima- und Umweltschutz sowie Digitalisierung, die Formulierung von Wirkungszielen, die Einführung von Wirkungsindikatoren zur Beurteilung der Zielerreichung oder Vorgaben zur Veröffentlichung von Beschreibungen der geförderten Vorhaben im Internet. Es wird empfohlen, die anderen in der gegenständlichen Untersuchung diskutierten Aspekte, vor allem bezüglich Additionalität und der Gründe für die vorzeitigen Beendigungen, im Auge zu behalten und in kommenden Untersuchungen aufzugreifen. Mit der erhofften Abschwächung der Pandemie sollten künftig auch längere Vergleichszeiträume zur Verfügung stehen. Die Anwendung der Sonderrichtlinien-Wirkungsindikatoren in der vorliegenden Evaluation kann als Ausgangspunkt für weiterführende Operationalisierungen genutzt werden, insbesondere mit Blick auf die Erfassung der Wirkungen des Moduls Prepare. 


\section{Tabellenverzeichnis}

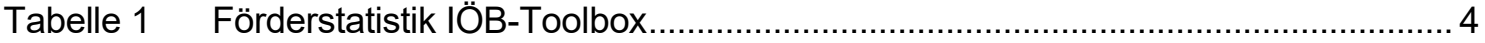

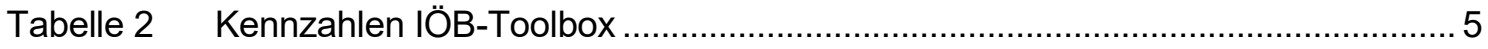

Tabelle 3 Online-Befragung: Übersicht Datenbasis und Rücklauf ................................... 9

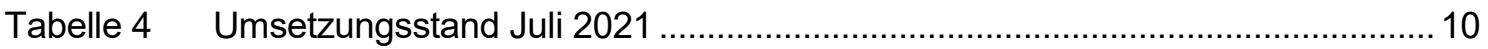

Tabelle $5 \quad$ Ziele und Wirkungsindikatoren (Impact-Dimension) .......................................... 17

\section{Abbildungsverzeichnis}

Abbildung 1 Relevanz verschiedener Informationskanäle (Transfer) ……….................... 11

Abbildung 2 Ziele, die mit der Beschaffung verfolgt wurden bzw. werden (Transfer) ........ 12

Abbildung 3 Probleme bei der geförderten Beschaffung (Transfer).................................. 13

Abbildung 4 Wirkungen mit Fokus auf den öffentlichen Beschaffer (Transfer).................. 15

Abbildung 5 Wirkungen mit Fokus auf den gesellschaftlichen Mehrwert (Transfer)........... 16

Abbildung 6 Verbesserung durch die Beschaffung im Vergleich zur Ausgangssituation... 18

Abbildung 7 Einschätzung des Förderverfahrens und der -abwicklung (Transfer)............. 19 


\section{JOANNEUM RESEARCH}

Forschungsgesellschaft mbH

POLICIES

Institut für Wirtschafts- und Innovationsforschung

Leonhardstrasse 59

8010 Graz

Tel: +433161488

$\mathrm{Fax}+433161480$

policies@joanneum.at

www.joanneum.at/policies

JOANNEUM RESEARCH

Forschungsgesellschaft mbH

POLICIES

Institut für Wirtschafts- und Innovationsforschung

Haus der Forschung

Sensengasse 1

Tel: +4315817520

Fax +43158175 20-2820

policies@joanneum.at

www.joanneum.at/policies

JOANNEUM RESEARCH

Forschungsgesellschaft mbH

POLICIES

Institut für Wirtschafts- und Innovationsforschung

Lakeside Science \& Technology Park

Lakeside B08a, EG

A-9020 Klagenfurt am Wörthersee

Tel.: +43 316 876-7553

Fax: +43 $3168769-7553$

policies@joanneum.at

www.joanneum.at/policies 